\title{
Dynamic Pricing and Inventory Control: Robust vs. Stochastic Uncertainty Models A Computational Study
}

\author{
Elodie Adida* and Georgia Perakis ${ }^{\dagger}$
}

August 2008, revised April 2009, December 2009

\begin{abstract}
In this paper, we consider a variety of models for dealing with demand uncertainty for a joint dynamic pricing and inventory control problem in a make-to-stock manufacturing system. We consider a multi-product capacitated, dynamic setting, where demand depends linearly on the price. Our goal is to address demand uncertainty using various robust and stochastic optimization approaches. For each of these approaches, we first introduce closed-loop formulations (adjustable robust and dynamic programming), where decisions for a given time period are made at the beginning of the time period, and uncertainty unfolds as time evolves. We then describe models in an open-loop setting, where decisions for the entire time horizon must be made at time zero. We conclude that the affine adjustable robust approach performs well (when compared to the other approaches such as dynamic programming, stochastic programming and robust open loop approaches) in terms of realized profits and protection against constraint violation while at the same time it is computationally tractable. Furthermore, we compare the complexity of these models and discuss some insights on a numerical example.
\end{abstract}

\footnotetext{
*Department of Mechanical and Industrial Engineering, University of Illinois at Chicago, elodie@uic.edu

${ }^{\dagger}$ Sloan School of Management, MIT, georgiap@mit.edu
} 


\section{Introduction, motivation and literature review}

Dynamic pricing, inventory control and revenue management have become very active research topics and have been extensively studied in the academic literature in Economics, Operations Management, and Marketing. In such settings, suppliers are maximizing their profits over a time horizon subject to some constraints, by adjusting their prices and production allocations. For example, the book by Porteus [40] and the book by Zipkin [51] review inventory management techniques, while the book by Talluri and van Ryzin [48] provides an overview of the revenue management and pricing literature. Elmaghraby and Keskinocak in [27] and the references therein provide a comprehensive literature review of dynamic pricing models while Bitran and Caldentey [18] provide an overview of research on dynamic pricing and its relation to Revenue Management.

In order to incorporate time-varying data, these problems are typically formulated as constrained dynamic optimization problems. The decision variables may thus vary over time. Many of the models in the process control and optimization literature assume an open-loop setting (see for example $[18,27]$ and the references therein). In such a setting, decisions for all time periods must be taken at the beginning of the time horizon. However, open-loop formulations have been criticized in management applications for not representing the setting realistically. Indeed, in most management decision-making problems, the system may be observed and decisions are made all along the time horizon based on the current state of the system. A closed-loop approach on the other hand allows feedback based on the observations made so far. When disturbances or uncertainty are present, in a closed-loop setting the decision-maker can adjust the decisions as time evolves. Solving a closed-loop model, as often done with Dynamic Programming, is significantly more complex than an open-loop model (especially if the model is nonlinear), and sometimes intractable because of the high dimension of the state space, unless the number of time periods is very small. Van Hessem and Bosgra [49] introduce a concept to solve a closed-loop constrained optimization problem over a reference trajectory and a feedback map. They formulate the model as a convex conic optimization problem and use interior-point methods. Balashevich et al. [6] develop numerical methods for the open-loop and closed-loop optimization of linear systems with discrete controls using a dual method. Federgruen and Heching [29] address pricing and inventory control for a single product under stochastic demand, with backorders, no fixed cost, and in a periodic review model. They characterize the value function and show that a base-stock policy is optimal. Chen and Simchi-Levi $([22,23])$ consider the model where ordering costs include a fixed cost component, both in the finite and infinite time horizon case. They show that if the time horizon is finite, an $(s, S, p)$ policy is optimal for additive demand, but not for multiplicative demand. Using $k$-convexity, they show that for an infinite horizon and input parameters independent of time, an $(s, S, p)$ policy is optimal for both types of demand models.

Open-loop and closed-loop models differ as soon as disturbances influence externally the system, or the data is uncertain. Recognizing the presence of uncertainty is essential for the practical applications of optimization. Ignoring uncertainty leads to solutions that may be very sub-optimal or even infeasible in practice. Stochastic models assume a specific probability distribution of the uncertain data. Dupačová [25] and Römisch and Schultz [41] investigated the sensitivity of the optimal solution with respect to the probability distribution, but the results are valid only for small perturbations, so knowing the probability distribution and its parameters remains critical. A variety of methods to address uncertainty in a stochastic way have been investigated over the last 
fifty years, including expectation optimization, minimization of deviations from a target, mini-max, maxi-min, and optimization over soft constraints. Sahinidis [43] reviews methods that have been developed in this area, from stochastic programming, fuzzy programming, to dynamic stochastic programming (in closed-loop setting). The books by Bertsekas and Tsitsiklis [10], Birge and Louveaux [17], and Zimmerman [50] provide more details.

In the last decade, some researchers have focused on treating uncertainty in an alternate way, known as robust optimization. This does not require to know the probability distribution of the uncertain data. Instead, they consider an uncertainty set for these data and look for a solution that performs well for any realization within this set. The uncertainty sets are based on risk preferences and tractability. See for example Soyster [47], Ben-Tal and Nemirovski [9], El-Ghaoui et al [26], Bertsimas and Sim [14] for more details. In a closed-loop setting for linear programs, Ben-Tal et al. [8] and Guslitzer [31] extend their robust optimization methodology to formulations where some variables may be determined after the realization of uncertain parameters. These variables are called adjustable. They address the difficulty of solving the adjustable robust counterpart problem by restricting adjustable variables to be affine functions of the uncertain data. The reader should refer to [1] for additional references.

The overall goal of this research is to introduce and study a joint dynamic pricing and inventory control problem in a monopoly setting. In particular, this paper considers an uncertain demand model that is linear in terms of the price. Additive and multiplicative uncertainty models are special cases of this demand model. Given the uncertain as well as dynamic nature of the problem, we consider a variety of possible approaches. We consider on one hand, closed-loop and (mainly for comparison purposes) open-loop approaches, and on the other hand, robust as well as stochastic optimization approaches. In the dynamic pricing application we consider in this paper, we establish that closed-loop formulations, while more realistic, are in general intractable both in a stochastic optimization framework as well as in a robust dynamic programming framework. Nevertheless, this paper presents a particular type of closed-loop model (through an affine adjustable robust approach) that (i) is tractable as compared to other possible closed-loop models, and (ii) performs well when compared with open-loop models in terms of realized profits and protection against constraint violation. Finally, we illustrate that, in contrast with robust optimization formulations, the performance of formulations based on stochastic optimization techniques is very sensitive to the accuracy of assumptions regarding the probability distribution of uncertain data.

In more detail we consider the following:

1. We consider both stochastic and robust optimization approaches. We study the advantages of each approach and illustrate the differences in the solutions they provide.

2. Within each of the two previous settings, we introduce closed-loop in addition to open-loop models and we verify the intuitive result that closed-loop models, while usually more realistic, are more complex to solve.

3. For each model, we provide an equivalent deterministic formulation: in the robust optimization framework, we obtain the robust counterpart reformulation while in the stochastic framework, we reformulate the problem assuming a particular probability distribution. (To ease exposition, we considered normal and uniform distributions, but our methodology can be extended to other probability distributions.) 
4. The models incorporate uncertainty not only in the objective function but also in the constraints. In the stochastic framework, we deal with constraint uncertainty by using the standard modelling tool of chance constraints. In the robust framework we determine the most adverse realization (given our attitude to uncertainty, i.e. our budget of uncertainty).

5. We compare in a numerical study the difficulty of solving the models we discuss and their performance, in terms of constraint violations, realized profits, and sensitivity to assumptions.

In Section 2, we describe the general setting of the problem. In Section 3, we introduce the closed-loop version of the problem and provide the adjustable robust formulation as well as the dynamic programming formulation. In Section 4, we detail an open-loop model both via robust optimization and stochastic optimization. In Section 5, we solve these models on a numerical example and compare the results.

\section{The Model}

We consider a supplier selling $N$ non perishable differentiated products, denoted $i=1, \ldots, N$. The time horizon $[0, T]$ is finite and time is viewed discretely $t=0,1, \ldots, T$. The supplier controls the price $p_{i}(t)$ and production rate $u_{i}(t)$ of each product at all times. We assume that no stockout is allowed. This might occur for example, when a contract between a supplier and a retailer does not allow delays in the delivery of the products, or when there is very high fixed backlog cost. Pekelman [38] studies a problem of deterministic optimal pricing and production for a single product with no backorders. Axsäter and Juntti [5] study echelon stock reorder policies with no backorders.

The inventory level at the beginning of a time period is a state variable, in the sense that its value at a given time depends on the decisions made from time zero up to that time period. The dynamics of the evolution of inventory is given by the following equation:

$$
I_{i}(t+1)=I_{i}(t)+u_{i}(t)-d_{i}(t), t=0, \ldots, T-1, \quad I_{i}(0)=I_{i}^{0}
$$

where $d_{i}(t)$ is the demand rate for product $i$ at time period $t$, and $I_{i}^{0}$ is the given initial inventory level for product $i$. Equivalently,

$$
I_{i}(t)=I_{i}^{0}+\sum_{s=0}^{t-1} u_{i}(s)-\sum_{s=0}^{t-1} d_{i}(s) .
$$

The revenue is given by

$$
\sum_{i=1}^{N} \sum_{t=0}^{T-1} p_{i}(t) d_{i}(t) .
$$

We assume that firms are profit maximizing.

In this model, multiple products share a single common production capacity $K(t)$. This assumption is a standard one in the literature that considers multiclass systems. For example, Bertsimas and Paschalidis [13] consider a multiclass make-to-stock system and assume that a single facility produces several products. Also in a make-to-stock manufacturing setting with multiple products, Kachani and Perakis [33] study this problem assuming that the total production capacity rate 
across all products is bounded. Gilbert [30] addresses the problem of jointly determining prices and production schedules for a set of items that are produced on the same production equipment and with a limited capacity. Maglaras and Meissner [36] consider a single firm that owns a fixed capacity of a resource that is consumed in the production of multiple products. Finally, Biller et al. [16] extend the single product model of dynamic pricing to cover supply chains with multiple products, each of which is assembled from a set of parts and shares common production capacity.

We assume moreover that the production costs are quadratic with the production rate with a coefficient $\gamma_{i}(t)$, and that the holding costs are linear with the inventory level, with coefficient $h_{i}(t)$. These types of costs have been used often in the literature on inventory control. Holt et al. [32] introduce a linear-quadratic inventory model in which the production and the holding costs are respectively the sum of a linear and a quadratic term in the production rate or the inventory rate. They justify this approximation for production costs from a connection with workforce costs. They observe that the cost of hiring and training people rises with the number hired, and the cost of laying off workers, including terminal pay, reorganization, etc., rises with the number laid off. Moreover, for fixed workforce, increasing production may incur overtime costs. Pindyck [39] models production costs for commodities such as copper, lumber and heating oil as quadratic costs. Finally, Sethi et al. [45] assume general convex production and inventory costs. Pekelman [38] studies a problem of optimal pricing and production for a single product with strictly convex production costs and linear holding costs. Clark and Scarf [24] introduce the Multi-Echelon Inventory Problem which includes linear holding costs. This model was used extensively in the literature.

\subsection{The Demand Model}

We assume that demand is linear with the price:

$$
d_{i}(t)=\alpha_{i}(t)-\beta_{i}(t) p_{i}(t) .
$$

In this equation, $\beta_{i}(t)$ represents the sensitivity of demand with respect to the price, and $\alpha_{i}(t)$ the part of the demand that is not related to the price. Notice that non negativity of the demand implies an upper bound on prices.

In this paper we assume that the demand for a product does not depend on the prices of other products. This assumption is standard in multi-product pricing problems when the products are considered distinct so that they target distinct classes of customers. Bertsimas and de Boer [11] study a joint pricing and resource allocation problem in which a finite supply of a resource can be used to produce multiple products and the demand for each product depends on its price. Paschalidis and Liu [37] consider a communication network with fixed routing that can accommodate multiple service classes and in which the arrival rate of a given class (or demand for that class) depends on the price per call of that class only. In their multi-product case, Biller et al. [16] assume that there are no diversions among products, i.e. that a change in the price for one product does not affect the demand for another product. They motivate this assumption by focusing on items that appeal to various consumer market segments, such as for example, luxury cars, SUVs, small pickups, etc. in the automotive industry.

Econometricians have considered the problem of estimating price elasticities over time. Senhadji and Montenegro [44] analyze time series to estimate short-run and long-run price elasticities 
via regression techniques. Slaughter [46] also considers elasticities that vary over time.

In the Operations Research literature, demand learning problems have motivated many researchers. In the case of models of demand linear with the price, the methods they propose can be applied to estimating the parameters $\alpha_{i}(),. \beta_{i}($.$) in our model. For example, Kachani, Perakis and Simon [34]$ design an approach that enable to achieve dynamic pricing while learning the price-demand linear relationship in an oligopoly. Caro and Gallien [19] investigate how demand learning effects impact the dynamic assortment of seasonal consumer goods. Araman and Caldentey [4] provide a solution method for dynamic pricing of non perishable product using Bayesian demand learning. Bertsimas and Mersereau [12] consider the adaptive learning of message effectiveness within a customer segment for interactive marketing. Chen and Plambeck [20] study the optimal stocking level of non perishable inventory when lost sales are not observed and the demand distribution must be learned.

Demand uncertainty results from uncertainty on parameters $\alpha_{i}(t)$ and $\beta_{i}(t)$. We denote

$$
\tilde{d}_{i}(t)=\tilde{\alpha}_{i}(t)-\tilde{\beta}_{i}(t) p_{i}(t)
$$

the realized demand, involving realized parameters $\tilde{\alpha}_{i}(t), \tilde{\beta}_{i}(t)$. In a setting with uncertainty, notice that the no stockout constraint is a non negativity constraint on the realized inventory level

$$
\tilde{I}_{i}(t)=I_{i}^{0}+\sum_{s=0}^{t-1} u_{i}(s)-\sum_{s=0}^{t-1}\left(\tilde{\alpha}_{i}(s)-\tilde{\beta}_{i}(s) p_{i}(s)\right)
$$

for $t=1, \ldots, T$. Similarly, realized holding costs involve the realized inventory levels, and realized profits involve realized demand.

\subsection{Two Approaches to the Dynamic Nature of the Problem}

\subsubsection{A Closed-loop Model}

In a closed-loop setting, decisions are made gradually as time unfolds. The firm may thus review her course of action as time evolves based on the observation of inventory levels and of realizations of past uncertain parameters. A closed-loop (or feedback) setting yields optimal policies that depend on time and state vectors. Note that a feedback solution may be approximated by deriving an open-loop solution and using rolling-horizon techniques.

Thus, at the beginning of time period $t$, inventory levels $I_{i}(t)$ are revealed as well as the realizations $\alpha_{i}(t), \beta_{i}(t)$, and the supplier decides $p_{i}(t), u_{i}(t)$ for all products. Let $\tilde{J}_{t}($.$) the (uncertain) profit-$ to-go from time period $t$, including the profit for time period $t$ and all remaining time periods. $\tilde{J}_{t}($.$) takes as argument the vector I(t)$ of current inventory level for all products. In a closed loop setting, at every time period the decision maker decides the price and production level of each product for this period in order to maximize profits for this time period as well as the rest of the 
time horizon. Thus we have

$$
\begin{aligned}
\tilde{J}_{t}(I(t))=\max _{p(t), u(t)} \quad & \sum_{i=1}^{N}\left(p_{i}(t)\left(\alpha_{i}(t)-\beta_{i}(t) p_{i}(t)\right)-\gamma_{i}(t) u_{i}(t)^{2}-h_{i}(t+1) I_{i}(t+1)\right)+\tilde{J}_{t+1}(I(t+1)) \\
\text { s.t. } \quad & \sum_{i=1}^{N} u_{i}(t) \leq K(t) \\
& p_{i}(t) \leq \frac{\alpha_{i}(t)}{\beta_{i}(t)}, \quad i=1, \ldots, N \\
& u_{i}(t), p_{i}(t) \geq 0, \quad i=1, \ldots, N \\
& I_{i}(t+1) \geq 0, \quad i=1, \ldots, N \\
& I_{i}(t+1) \equiv I_{i}(t)+u_{i}(t)-\alpha_{i}(t)+\beta_{i}(t) p_{i}(t), \quad i=1, \ldots, N .
\end{aligned}
$$

Notice that the profit-to-go from the next time period $\tilde{J}_{t+1}(I(t+1))$ is uncertain, since the demand parameters of future time periods are uncertain, and thus the profit-to-go from time $t$ is also uncertain. However, the current parameters are revealed, so the profit generated by the end of the current time period is deterministic. Moreover, constraints may be satisfied deterministically.

\subsubsection{An Open-loop Model}

In an open-loop setting, all decisions $p_{i}(t), u_{i}(t), t=0, \ldots, T-1, i=1, \ldots, N$ are made in the beginning of the time horizon (that is, at time zero). In other words, the firm makes an irreversible commitment to a future course of action. As a result, this is a "static" approach. This situation may arise in practice if a contract with buyers or with a labor union forces the firm to commit to prices or workforce at the beginning of the time horizon. In such a setting, the decisions depend on time and the initial state vector only, the supplier does not use any other information such as current state variable or updated estimate of uncertain data.

In an open-loop setting, the decision-maker thus must decide at time zero the prices and production levels for all products at all times, with the goal of maximizing the profits over the entire time horizon from all products. The decision maker thus faces the following optimization problem, which involves some uncertain quantities:

$$
\begin{aligned}
\max _{p(.), u(.),} & \sum_{i=1}^{N} \sum_{t=0}^{T-1}\left(p_{i}(t)\left(\tilde{\alpha}_{i}(t)-\tilde{\beta}_{i}(t) p_{i}(t)\right)-\gamma_{i}(t) u_{i}(t)^{2}-h_{i}(t+1) \tilde{I}_{i}(t+1)\right) \\
\text { s.t. } & \sum_{i=1}^{N} u_{i}(t) \leq K(t), \quad t=0, \ldots, T-1 \\
& \tilde{I}_{i}(t) \geq 0, \quad t=1, \ldots, T, \quad i=1, \ldots, N \\
& p_{i}(t) \leq \frac{\tilde{\alpha}_{i}(t)}{\tilde{\beta}_{i}(t)}, \quad t=0, \ldots, T-1, \quad i=1, \ldots, N \\
& u_{i}(t), p_{i}(t) \geq 0, \quad t=0, \ldots, T-1, \quad i=1, \ldots, N \\
& \tilde{I}_{i}(t) \equiv I_{i}^{0}+\sum_{s=0}^{t-1} u_{i}(s)-\sum_{s=0}^{t-1}\left(\tilde{\alpha}_{i}(s)-\tilde{\beta}_{i}(s) p_{i}(s)\right), \quad t=1, \ldots, T, \quad i=1, \ldots, N .
\end{aligned}
$$

Notice that if all parameters were deterministic, the problem would be a convex quadratic program with linear constraints, with $2 N T$ control variables. 


\subsection{Approaches to Demand Uncertainty}

\subsubsection{Robust Optimization}

In robust optimization, we aim at protecting the system against unknown but bounded disturbances:

$$
\tilde{\alpha}_{i}(t) \in\left[\alpha_{i}(t)-\hat{\alpha}_{i}(t), \alpha_{i}(t)+\hat{\alpha}_{i}(t)\right], \quad \tilde{\beta}_{i}(t) \in\left[\beta_{i}(t)-\hat{\beta}_{i}(t), \beta_{i}(t)+\hat{\beta}_{i}(t)\right],
$$

where $\hat{\alpha}_{i}(t), \hat{\beta}_{i}(t)$ are some given half-length of ranges for the realizations. To avoid making assumptions that are difficult to satisfy in practice, we do not introduce a specific probability distribution. However, it seems overly conservative to protect the solution against a systematic worst-case scenario (typically, value at an extreme of the range) at all times, or for all products, or for all parameters, since such scenarios are highly unlikely to occur. Therefore, we attempt to take advantage of risk pooling by guaranteeing feasibility of the solution for any realization within some uncertainty set and maximizing the worst case profit within this set.

Motivated by this observation, we introduce "budget of uncertainty" functions as an efficient way to measure the trade-off between conservativeness and performance. The budget of uncertainty further restricts the allowed realizations of data within the range around the nominal values given above in 1 . The budget of uncertainty is input in the model. A risk averse decision maker takes a conservative approach by considering large uncertainty sets (large budget of uncertainty) in order to be protected for any realization within this set. A risk taking decision maker sacrifices immunity for performance and chooses smaller uncertainty sets. The risk aversion may depend for instance on true preferences, confidence in data estimates, stakes of the decisions, etc.

Uncertainty set $\mathcal{F}$ is the set of realizations that satisfy the constraints of budget of uncertainty and allowed range 1 (set of feasible realizations).

There exist a variety of ways to model these budget constraints. In this paper, we view budgets as taken across parameters:

$$
\left|z_{i}(t)\right|+\left|y_{i}(t)\right| \leq \Gamma_{i}(t) \quad \forall t, i
$$

where

$$
z_{i}(t)=\frac{\tilde{\alpha}_{i}(t)-\alpha_{i}(t)}{\hat{\alpha}_{i}(t)}, \quad y_{i}(t)=\frac{\tilde{\beta}_{i}(t)-\beta_{i}(t)}{\hat{\beta}_{i}(t)}
$$

i.e. $z_{i}(t)$ (resp. $\left.y_{i}(t)\right)$ is the scaled deviation of the realized value $\tilde{\alpha}_{i}(t)$ (resp. $\tilde{\beta}_{i}(t)$ ) from the nominal value $\alpha_{i}(t)$ (resp. $\left.\beta_{i}(t)\right)$. Note that the allowed ranges 1 for $\tilde{\alpha}_{i}(t)$ and $\tilde{\beta}_{i}(t)$ imply $z_{i}(t), y_{i}(t) \in$ $[-1,1]$.

This model captures that at a given time and for a given product, it is unlikely that both parameters take the worst value. This constraint separates across time and across products, but it binds the parameters together. In constraints that are linear in the parameters (such as the no stockout constraints), linearity will be preserved. But in constraints such as the upper bound on prices that involve expression $\frac{\tilde{\alpha}_{i}(t)}{\tilde{\beta}_{i}(t)}$ that is nonlinear in the parameters determining the robust counterpart implies solving a nonlinear optimization problem. 


\subsubsection{Stochastic Optimization}

In stochastic optimization, the probability distribution of uncertain parameters $\tilde{\alpha}_{i}(t), \tilde{\beta}_{i}(t)$ is assumed to be known, with respective mean $\alpha_{i}(t), \beta_{i}(t)$ and standard deviation $\frac{\hat{\alpha}_{i}(t)}{2}, \frac{\hat{\beta}_{i}(t)}{2}$. This ensures that the robust and stochastic models are consistent, in the sense that the range of variation corresponds to two standard deviations around the mean. In general, the goal is to maximize the expected profits. One way to deal with constraints that involve uncertain data is to introduce a given tolerance $\epsilon$ for constraint violation, such that a given constraint must be satisfied with probability $1-\epsilon$. Variations of this type include a tolerance for simultaneous violations. The complexity of these formulations essentially depends on the interdependence of constraints, the specific probability distributions, and the difficulty of evaluating the expected profits and the probability of violations under this distributional assumption.

\subsubsection{Deterministic Optimization}

As a tool of comparison, we will implement the solution obtained when ignoring that demand may be uncertain. The uncertain parameters are then assumed to be deterministically equal to the nominal value (or mean) throughout the time horizon. In numerical experiments, we will generate random realizations and observe how this solution performs.

\subsubsection{Perfect Hindsight}

The perfect hindsight solution provides another point of comparison. It is obtained by assuming that the randomly generated realizations are known in advance. The corresponding optimal solution is then computed based on this knowledge.

Notice that as no uncertainty is present in the deterministic and perfect hindsight approach, closed- and open-loop settings are equivalent.

\section{Closed-loop Problem}

\subsection{Dynamic Programming}

In a closed-loop setting where probability distributions are given, using Bellman's equation, the dynamic programming problem to solve at time $t$ is given by

$$
\begin{aligned}
J_{t}(I(t))=\max _{u(t), p(t)} & \sum_{i=1}^{N} p_{i}(t)\left(\alpha_{i}(t)-\beta_{i}(t) p_{i}(t)\right)-\gamma_{i}(t) u_{i}(t)^{2}-h_{i}(t+1) I_{i}(t+1)+E\left[\tilde{J}_{t+1}(I(t+1))\right] \\
\text { such that } \quad & I_{i}(t+1) \equiv I_{i}(t)+u_{i}(t)-\alpha_{i}(t)+\beta_{i}(t) p_{i}(t) \quad \forall i \\
& \sum_{i=1}^{N} u_{i}(t) \leq K(t) \\
& p_{i}(t) \leq \frac{\alpha_{i}(t)}{\beta_{i}(t)} \forall i \\
& u_{i}(t), p_{i}(t), I_{i}(t+1) \geq 0 \quad \forall i
\end{aligned}
$$

where $\tilde{J}_{t}(I(t))$ is obtained by substituting above respectively $\tilde{\alpha}_{i}(t), \tilde{\beta}_{i}(t), \tilde{I}_{i}(t+1)$ for $\alpha_{i}(t), \beta_{i}(t)$, $I_{i}(t+1)$. Evaluating the expectation in the objective is a difficult task in general. Not only it is 
not obvious how to express the profit-to-go from the next time period resulting from the current decisions, but also calculating its expectation can be difficult if it is nonlinear, depending on the given probability distribution.

\subsubsection{Illustration: the 2-period, one product case with parameters normally dis- tributed}

We illustrate the solution method on the 2-period, one product case. Let

$$
\begin{aligned}
& q^{A}(I(1))=\operatorname{Pr}\left(I(1) \geq \frac{\tilde{\alpha}(1)+h(2) \tilde{\beta}(1)}{2}\right) \\
& q^{B}(I(1))=\operatorname{Pr}\left(\frac{\tilde{\alpha}(1)}{2}<I(1)<\frac{\tilde{\alpha}(1)+h(2) \tilde{\beta}(1)}{2}\right) \\
& q^{C}(I(1))=\operatorname{Pr}\left(\frac{\tilde{\alpha}(1)}{2}-K(1)(1+\gamma(1) \tilde{\beta}(1)) \leq I(1) \leq \frac{\tilde{\alpha}(1)}{2}\right) \\
& q^{D}(I(1))=\operatorname{Pr}\left(0 \leq I(1)<\frac{\tilde{\alpha}(1)}{2}-K(1)(1+\gamma(1) \tilde{\beta}(1))\right)
\end{aligned}
$$

Calculating these probabilities involves convoluting $\tilde{\alpha}(t)$ and $\tilde{\beta}(t)$.

To ease exposition, we consider the example of Normal distributions as is often the case in the literature (see for example [7], [28], [35], [42]):

$$
\tilde{\alpha}_{i}(t) \sim \mathcal{N}\left(\alpha_{i}(t), \frac{\hat{\alpha}_{i}(t)}{2}\right), \quad \tilde{\beta}_{i}(t) \sim \mathcal{N}\left(\beta_{i}(t), \frac{\hat{\beta}_{i}(t)}{2}\right)
$$

(Note that in the numerical study at the end of this paper, we also considered uniform distributions for the uncertain data, as in [42].) For Normal distributions, the results are highly nonlinear in the argument:

$$
\begin{aligned}
q^{A}(I(1)) & =\varphi\left(\frac{2 I(1)-\alpha(1)-h(2) \beta(1)}{\sqrt{\frac{\hat{\alpha}(1)^{2}}{4}+h(2)^{2} \frac{\hat{\beta}(1)^{2}}{4}}}\right) \\
q^{B}(I(1)) & =\int_{-\infty}^{2 I(1)}\left(1-\varphi\left(\frac{2 I(1)-x-h(2) \beta(1)}{h(2) \frac{\hat{\beta}(1)}{2}}\right)\right) f(x) d x \\
q^{C}(I(1)) & =\int_{2 I(1)}^{\infty}\left(1-\varphi\left(\frac{x-2 I(1)-2 K(1)(1+\gamma(1) \beta(1))}{K(1) \gamma(1) \hat{\beta}(1)}\right)\right) f(x) d x \\
q^{D}(I(1)) & =1-\varphi\left(\frac{2 I(1)-\alpha(1)+2 K(1)(1+\gamma(1) \beta(1))}{\sqrt{\frac{\hat{\alpha}(1)^{2}}{4}+K(1)^{2} \gamma(1)^{2} \hat{\beta}(1)^{2}}}\right)
\end{aligned}
$$

where $\tilde{\alpha}(1)$ has probability density function $f($.$) .$ 
Then, using Table 1 ,

$$
\begin{aligned}
E\left[\tilde{J}_{1}(I(1))\right] & =q^{A}(I(1)) e^{A}(I(1))+q^{B}(I(1)) e^{B}(I(1))+q^{C}(I(1)) e^{C}(I(1))+q^{D}(I(1)) e^{D}(I(1)) \\
e^{A}(I(1)) & =v E\left[\frac{1}{\tilde{\beta}(1)}\right]+\frac{h(2) \alpha(1)}{2}+\frac{h(2)^{2} \beta(1)}{4}-h(2) I(1) \\
e^{B}(I(1)) & =I(1)(\alpha(1)-I(1)) E\left[\frac{1}{\tilde{\beta}(1)}\right] \\
e^{C}(I(1)) & =v E\left[\frac{1}{\tilde{\beta}(1)}\right]+\gamma(1) E\left[\frac{1}{1+\gamma(1) \tilde{\beta}(1)}\right]\left(-v+\frac{\alpha(1) I(1)}{2}-I(1)^{2}\right) \\
e^{D}(I(1)) & =E\left[\frac{1}{\tilde{\beta}(1)}\right](K(1)+I(1))(\alpha(1)-K(1)-I(1))-\gamma(1) K(1)^{2}
\end{aligned}
$$

where

$$
v \equiv \frac{\alpha(1)^{2}+\operatorname{Var}(\tilde{\alpha}(1))}{4} .
$$

We notice that these expressions are linear or quadratic in the argument $I(1)$. Therefore, the resulting problem to solve in the first stage is highly nonlinear via its objective function. Assuming uniform distributions also leads to a complex nonlinear formulation.

\subsection{Adjustable Robust Optimization}

\subsubsection{Formulation}

In the adjustable setting, a decision at time period $t$ can be adjusted after realizations of data from time 0 to $t-1$ are revealed. At the beginning of time period $t, \alpha(t), \beta(t)$ are revealed and inventory levels $I(t)$ are observed. In particular, the inventory levels at the next step $I(t+1)$ and the constraints to satisfy at this period are deterministic. Future parameters are still uncertain, therefore the profit-to-go from $t+1$ is uncertain. In a robust approach, we aim at determining feasible decision variables for the current time period that maximize worst case profit-to-go within the uncertainty sets. Thus at time $t$, the profit-to-go is

$$
\begin{aligned}
J_{t}(I(t))=\max _{u(t), p(t), Q} \quad & \\
\text { s.t. } \quad & Q \leq \sum_{i=1}^{N} p_{i}(t)\left(\alpha_{i}(t)-\beta_{i}(t) p_{i}(t)\right)-\gamma_{i}(t) u_{i}(t)^{2}-h_{i}(t+1) I_{i}(t+1) \\
& \quad+\tilde{J}_{t+1}(I(t+1)) \quad \forall(\tilde{\alpha}(t+1), \tilde{\beta}(t+1)) \in \mathcal{F}(t+1) \\
& I_{i}(t+1) \equiv I_{i}(t)+u_{i}(t)-\alpha_{i}(t)+\beta_{i}(t) p_{i}(t) \quad \forall i \\
& \sum_{i=1}^{N} u_{i}(t) \leq K(t) \\
& p_{i}(t) \leq \frac{\alpha_{i}(t)}{\beta_{i}(t)} \quad \forall i \\
& u_{i}(t), p_{i}(t), I_{i}(t+1) \geq 0 \quad \forall i
\end{aligned}
$$

where $\tilde{J}_{t}(I(t))$ is obtained by substituting above respectively $\tilde{\alpha}_{i}(t), \tilde{\beta}_{i}(t), \tilde{I}_{i}(t+1)$ for $\alpha_{i}(t), \beta_{i}(t)$, $I_{i}(t+1)$. 


\subsubsection{Adjustable robust counterpart problem}

In order to obtain a deterministic equivalent problem (adjustable robust counterpart), we solve as a function of $I(t+1)$ :

$$
\min _{(\tilde{\alpha}(t+1), \tilde{\beta}(t+1)) \in \mathcal{F}(t+1)} \tilde{J}_{t+1}(I(t+1)) .
$$

Then the robust counterpart problem is given by:

$$
\begin{aligned}
J_{t}(I(t))=\max _{u(t), p(t)} \quad & \sum_{i=1}^{N} p_{i}(t)\left(\alpha_{i}(t)-\beta_{i}(t) p_{i}(t)\right)-\gamma_{i}(t) u_{i}(t)^{2}-h_{i}(t+1) I_{i}(t+1) \\
\text { s.t. } \quad \min _{(\tilde{\alpha}(t+1), \tilde{\beta}(t+1)) \in \mathcal{F}(t+1)} \tilde{J}_{t+1}(I(t+1)) & I_{i}(t+1) \equiv I_{i}(t)+u_{i}(t)-\alpha_{i}(t)+\beta_{i}(t) p_{i}(t) \quad \forall i \\
& \sum_{i=1}^{N} u_{i}(t) \leq K(t) \\
& p_{i}(t) \leq \frac{\alpha_{i}(t)}{\beta_{i}(t)} \forall i \\
& u_{i}(t), p_{i}(t), I_{i}(t+1) \geq 0 \quad \forall i
\end{aligned}
$$

\begin{tabular}{|c|c|c|c|c|}
\hline & $u(1)$ & $p(1)$ & $I(2)$ & $J_{1}(I(1))$ \\
\hline$I(1) \geq \frac{\alpha(1)+h(2) \beta(1)}{2}$ & 0 & $\frac{\alpha(1)-h(2) \beta(1)}{2 \beta(1)}$ & $I(1)-\frac{\alpha(1)+h(2) \beta(1)}{2}$ & $\frac{(\alpha(1)+h(2) \beta(1))^{2}}{4 \beta(1)}-h(2) I(1)$ \\
\hline$\frac{\alpha(1)}{2} \leq I(1) \leq \frac{\alpha(1)+h(2) \beta(1)}{2}$ & 0 & $\frac{\alpha(1)-I(1)}{\beta(1)}$ & 0 & $\frac{I(1)(\alpha(1)-I(1))}{\beta(1)}$ \\
\hline$\frac{\alpha(1)}{2}-K(1)(1+\gamma(1) \beta(1)) \leq I(1) \leq \frac{\alpha(1)}{2}$ & $\frac{\alpha(1)-2 I(1)}{2(1+\gamma(1) \beta(1))}$ & $\frac{\alpha(1)}{2 \beta(1)}+\frac{\alpha(1)-2 I(1)}{2\left(\beta(1)+\frac{1}{\gamma(1)}\right)}$ & 0 & $\frac{\alpha(1)^{2}}{4 \beta(1)}-\frac{\gamma(1)(\alpha(1)-2 I(1))^{2}}{4(1+\gamma(1) \beta(1))}$ \\
\hline $0 \leq I(1) \leq \frac{\alpha(1)}{2}-K(1)(1+\gamma(1) \beta(1))$ & $K(1)$ & $\frac{\alpha(1)-(K(1)+I(1))}{\beta(1)}$ & 0 & $\begin{array}{c}\frac{(K(1)+I(1))(\alpha(1)-K(1)-I(1))}{\beta(1)} \\
-\gamma(1) K(1)^{2}\end{array}$ \\
\hline
\end{tabular}

\subsubsection{Illustration: the 2-period, one product case}

We will illustrate for the 2-period, one product case introduced in Section 3.1.1. At time 1, the supplier solves the following problem:

$$
\begin{aligned}
J_{1}(I(1))=\max _{u(1), p(1), I(2)} & p(1)(\alpha(1)-\beta(1) p(1))-\gamma(1) u(1)^{2}-h(2) I(2) \\
\text { such that } & I(2) \equiv I(1)+u(1)-\alpha(1)+\beta(1) p(1) \geq 0 \\
& 0 \leq p(1) \leq \frac{\alpha(1)}{\beta(1)} \\
& 0 \leq u(1) \leq K(1)
\end{aligned}
$$

After introducing Lagrange multipliers, we obtain the solution for problem (2) in Table 1.

Table 1: Optimal policies for the second stage problem (2) 
We then solve four corresponding subproblems:

$$
\begin{aligned}
& G^{A}(I(1))=\min _{y, z} \quad \frac{(\alpha(1)+z \hat{\alpha}(1)+h(2)(\beta(1)+y \hat{\beta}(1)))^{2}}{4(\beta(1)+y \hat{\beta}(1))}-h(2) I(1) \\
& \text { s.t. } \quad-1 \leq y, z \leq 1, \quad|y|+|z| \leq \Gamma(1) \\
& I(1) \geq \frac{\alpha(1)+z \hat{\alpha}(1)+h(2)(\beta(1)+y \hat{\beta}(1))}{2} \\
& G^{B}(I(1))=\min _{y, z} \quad \frac{I(1)(\alpha(1)+z \hat{\alpha}(1)-I(1))}{\beta(1)+y \hat{\beta}(1)} \\
& \text { s.t. } \quad-1 \leq y, z \leq 1, \quad|y|+|z| \leq \Gamma(1) \\
& \frac{\alpha(1)+z \hat{\alpha}(1)}{2} \leq I(1) \leq \frac{\alpha(1)+z \hat{\alpha}(1)+h(2)(\beta(1)+y \hat{\beta}(1))}{2} \\
& G^{C}(I(1))=\min _{y, z} \quad \frac{(\alpha(1)+z \hat{\alpha}(1))^{2}}{4(\beta(1)+y \hat{\beta}(1))}-\frac{\gamma(1)(\alpha(1)+z \hat{\alpha}(1)-2 I(1))^{2}}{4(1+\gamma(1)(\beta(1)+y \hat{\beta}(1)))} \\
& \text { s.t. } \quad-1 \leq y, z \leq 1, \quad|y|+|z| \leq \Gamma(1) \\
& \frac{\alpha(1)+z \hat{\alpha}(1)}{2}-K(1)(1+\gamma(1)(\beta(1)+y \hat{\beta}(1))) \leq I(1) \leq \frac{\alpha(1)+z \hat{\alpha}(1)}{2} \\
& G^{D}(I(1))=\min _{y, z} \quad \frac{(K(1)+I(1))(\alpha(1)+z \hat{\alpha}(1)-K(1)-I(1))}{\beta(1)+y \hat{\beta}(1)}-\gamma(1) K(1)^{2} \\
& \text { s.t. } \quad-1 \leq y, z \leq 1, \quad|y|+|z| \leq \Gamma(1) \\
& I(1) \leq \frac{\alpha(1)+z \hat{\alpha}(1)}{2}-K(1)(1+\gamma(1)(\beta(1)+y \hat{\beta}(1)))
\end{aligned}
$$

We observe that these subproblems involve linear constraints and a nonlinear objective (quotient of terms linear or quadratic in the decision variables). The structure of these subproblems depends on the type of budget of uncertainty chosen. The objective is nonlinear in this example because the budget of uncertainty is shared by the variables $\tilde{\alpha}_{i}(t)$ and $\tilde{\beta}_{i}(t)$. Then the first stage problem is:

$$
\begin{aligned}
J_{0}\left(I^{0}\right)=\max _{u(0), p(0), I(1)} & p(0)(\alpha(0)-\beta(0) p(0))-\gamma(0) u(0)^{2}-h(1) I(1) \\
& +\min \left\{G^{A}(I(1)), G^{B}(I(1)), G^{C}(I(1)), G^{D}(I(1))\right\} \\
\text { such that } & I(1) \equiv I^{0}+u(0)-\alpha(0)+\beta(0) p(0) \geq 0 \\
& 0 \leq p(0) \leq \frac{\alpha(0)}{\beta(0)} \\
& 0 \leq u(0) \leq K(0)
\end{aligned}
$$

This problem has linear constraints and a nonlinear objective, whose structure depends on the type of budget of uncertainty constraint.

\subsection{Affine Adjustable Robust Optimization}

\subsubsection{Formulation}

The model described above may become significantly complex for multiple time periods because of the "curse of dimensionality". Analogously, the dynamic programming formulation we detail 
in Section 3.1 suffers from the same issue in practice. In this section, we consider the subclass of controls that are linear combinations of the uncertain realizations of the demand parameters. This solution is suboptimal since we restrict the possibilities of available decisions. However, researchers have shown that optimizing over this subclass of controls yields a good approximation of the optimal solution, while providing a tractable model (see Ben-Tal et al. [8], Guslitzer [31], Chen, Sim and Sun [21]).

In such an approximation, we want to find the price and production at time $t$ that are linear combinations of the demand parameters at time $t$ that have just been realized and observed:

$$
\begin{aligned}
& u_{i}(t)=u_{i}^{0}(t)+\delta_{i}(t) \alpha_{i}(t)+\eta_{i}(t) \beta_{i}(t) \\
& p_{i}(t)=p_{i}^{0}(t)+\theta_{i}(t) \alpha_{i}(t)+\rho_{i}(t) \beta_{i}(t)
\end{aligned}
$$

where $X=\left(u_{i}^{0}(t), p_{i}^{0}(t), \delta_{i}(t), \eta_{i}(t), \theta_{i}(t), \rho_{i}(t), i=1 \ldots, N, t=0 \ldots, T-1\right)$ must be determined at time zero. We notice that the number of variables has increased from $2 N T$ (price and production rate of each product at each time) to $6 N T$ (the three coefficients of the linear relationships above for the price and the production rate).

Since the controls $u_{i}(t), p_{i}(t)$ depend on the uncertain parameters $\tilde{\alpha}_{i}(t), \tilde{\beta}_{i}(t)$, they are uncertain as well until the beginning of time period $t$ when the demand is realized and the demand parameters are revealed. In this sense, the approach is adjustable because the future price and production rates are adjusted based on the future realization of the demand parameters. However, the optimization problem is solved at time zero. Therefore, even though there are more variables and more subproblems, the dimension of this formulation does not increase exponentially with the number of time periods, as it does in the DP and the adjustable robust approaches.

Thus, to determine at time zero the $6 N T$ coefficients, we solve (for $Q$ and $X$, where $X=$ $\left.\left(u_{i}^{0}(t), p_{i}^{0}(t), \delta_{i}(t), \eta_{i}(t), \theta_{i}(t), \rho_{i}(t), i=1 \ldots, N, t=0 \ldots, T-1\right)\right)$

$$
\begin{array}{ll}
\max _{X, Q} & Q \\
\text { s.t. } & Q \leq \sum_{i=1}^{N} \sum_{t=0}^{T-1} \tilde{p}_{i}(t)\left(\tilde{\alpha}_{i}(t)-\tilde{\beta}_{i}(t) \tilde{p}_{i}(t)\right)-\gamma_{i}(t) \tilde{u}_{i}(t)^{2}-h_{i}(t+1) \tilde{I}_{i}(t+1) \quad \forall(\tilde{\alpha}(.), \tilde{\beta}(.)) \in \mathcal{F}(.) \\
& \tilde{I}_{i}(t) \equiv I_{i}^{0}+\sum_{s=0}^{t-1} \tilde{u}_{i}(s)-\sum_{s=0}^{t-1}\left(\tilde{\alpha}_{i}(s)-\tilde{\beta}_{i}(s) \tilde{p}_{i}(s)\right) \quad \forall i, t \\
& \tilde{u}_{i}(t) \equiv u_{i}^{0}(t)+\delta_{i}(t) \tilde{\alpha}_{i}(t)+\eta_{i}(t) \tilde{\beta}_{i}(t) \quad \forall i, t \\
& \tilde{p}_{i}(t) \equiv p_{i}^{0}(t)+\theta_{i}(t) \tilde{\alpha}_{i}(t)+\rho_{i}(t) \tilde{\beta}_{i}(t) \quad \forall i, t \\
& \sum_{i=1}^{N} \tilde{u}_{i}(t) \leq K(t) \quad \forall(\tilde{\alpha}(t), \tilde{\beta}(t)) \in \mathcal{F}(t), \quad \forall t \\
& \tilde{p}_{i}(t) \leq \frac{\tilde{\alpha}_{i}(t)}{\tilde{\beta}_{i}(t)} \quad \forall\left(\tilde{\alpha}_{i}(t), \tilde{\beta}_{i}(t)\right) \in \mathcal{F}_{i}(t), \quad \forall i, t, \\
& \tilde{u}_{i}(t), \tilde{p}_{i}(t) \geq 0 \quad \forall\left(\tilde{\alpha}_{i}(t), \tilde{\beta}_{i}(t)\right) \in \mathcal{F}_{i}(t), \quad \forall i, t \\
& \tilde{I}_{i}(t+1) \geq 0, \quad \forall\left(\tilde{\alpha}_{i}(s), \tilde{\beta}_{i}(s)\right) \in \mathcal{F}_{i}(s), \quad s=0, \ldots, t \quad \forall i, t
\end{array}
$$


which can be equivalently formulated as

$$
\begin{array}{ll}
\max _{X, Q} \quad & \\
\text { s.t. } \quad & Q \leq \sum_{i=1}^{N} \sum_{t=0}^{T-1}\left(p_{i}^{0}(t)+\theta_{i}(t) \tilde{\alpha}_{i}(t)+\rho_{i}(t) \tilde{\beta}_{i}(t)\right)\left(\tilde{\alpha}_{i}(t)-\tilde{\beta}_{i}(t)\left(p_{i}^{0}(t)+\theta_{i}(t) \tilde{\alpha}_{i}(t)+\rho_{i}(t) \tilde{\beta}_{i}(t)\right)\right) \\
& -\gamma_{i}(t)\left(u_{i}^{0}(t)+\delta_{i}(t) \tilde{\alpha}_{i}(t)+\eta_{i}(t) \tilde{\beta}_{i}(t)\right)^{2}-h_{i}(t+1) \tilde{I}_{i}(t+1) \quad \forall(\tilde{\alpha}(.), \tilde{\beta}(.)) \in \mathcal{F}(.) \quad(3) \\
& \tilde{I}_{i}(t) \equiv I_{i}^{0}+\sum_{s=0}^{t-1}\left(u_{i}^{0}(s)+\delta_{i}(s) \tilde{\alpha}_{i}(s)+\eta_{i}(s) \tilde{\beta}_{i}(s)\right) \\
& -\sum_{s=0}^{t-1}\left(\tilde{\alpha}_{i}(s)-\tilde{\beta}_{i}(s)\left(p_{i}^{0}(s)+\theta_{i}(s) \tilde{\alpha}_{i}(s)+\rho_{i}(s) \tilde{\beta}_{i}(s)\right)\right) \quad \forall i, t \\
& \sum_{i=1}^{N}\left(u_{i}^{0}(t)+\delta_{i}(t) \tilde{\alpha}_{i}(t)+\eta_{i}(t) \tilde{\beta}_{i}(t)\right) \leq K(t) \quad \forall(\tilde{\alpha}(t), \tilde{\beta}(t)) \in \mathcal{F}(t), \quad \forall t \\
& p_{i}^{0}(t)+\theta_{i}(t) \tilde{\alpha}_{i}(t)+\rho_{i}(t) \tilde{\beta}_{i}(t) \leq \frac{\tilde{\alpha}_{i}(t)}{\tilde{\beta}_{i}(t)} \forall\left(\tilde{\alpha}_{i}(t), \tilde{\beta}_{i}(t)\right) \in \mathcal{F}_{i}(t), \quad \forall i, t \\
& u_{i}^{0}(t)+\delta_{i}(t) \tilde{\alpha}_{i}(t)+\eta_{i}(t) \tilde{\beta}_{i}(t) \geq 0 \quad \forall\left(\tilde{\alpha}_{i}(t), \tilde{\beta}_{i}(t)\right) \in \mathcal{F}_{i}(t), \quad \forall i, t \\
& p_{i}^{0}(t)+\theta_{i}(t) \tilde{\alpha}_{i}(t)+\rho_{i}(t) \tilde{\beta}_{i}(t) \geq 0 \quad \forall\left(\tilde{\alpha}_{i}(t), \tilde{\beta}_{i}(t)\right) \in \mathcal{F}_{i}(t), \quad \forall i, t \\
& \tilde{I}_{i}(t+1) \geq 0 \quad \forall\left(\tilde{\alpha}_{i}(s), \tilde{\beta}_{i}(s)\right) \in \mathcal{F}_{i}(s), s=0 \ldots, t, \quad \forall i, t
\end{array}
$$

In the next subsection, we write this problem deterministically by finding, independently for each constraint, the worst realization within all feasible realizations. the equivalent deterministic problem is called robust counterpart.

\subsubsection{Affine Adjustable Robust Counterpart Problem (AARC)}

We can obtain a deterministic equivalent problem by finding, for each constraint involving uncertain parameters (i.e. constraints $(3),(4),(5),(6),(7)$ and (8)), the feasible realization that makes the constraint the most difficult to satisfy. For example, for constraint (4), we have to determine the realization $(\tilde{\alpha}(t), \tilde{\beta}(t)) \in \mathcal{F}(t)$ that maximizes the left hand side of the inequality.

Theorem 1. The affine adjustable robust counterpart problem (AARC) is the following:

$$
\begin{array}{ll}
\max _{X} & \sum_{i=1}^{N} \sum_{t=0}^{T-1} M_{i}(t, X) \\
\text { s.t. } & \sum_{i=1}^{N}\left(u_{i}^{0}(t)+\delta_{i}(t) \alpha_{i}(t)+\eta_{i}(t) \beta_{i}(t)+A_{i}\left(t, \delta_{i}(t), \eta_{i}(t)\right) \leq K(t) \quad \forall t\right. \\
& C_{i}(t, X) \leq 0 \quad \forall i, t \\
& u_{i}^{0}(t)+\delta_{i}(t) \alpha_{i}(t)+\eta_{i}(t) \beta_{i}(t)-A_{i}\left(t, \delta_{i}(t), \eta_{i}(t)\right) \geq 0 \quad \forall i, t \\
& p_{i}^{0}(t)+\theta_{i}(t) \alpha_{i}(t)+\rho_{i}(t) \beta_{i}(t)-A_{i}\left(t, \theta_{i}(t), \rho_{i}(t)\right) \geq 0 \quad \forall i, t \\
& B_{i}(t, X) \geq 0 \quad \forall i, t
\end{array}
$$


where

$$
\begin{aligned}
& m_{i}(t, X, y, z)=-H_{i}(t+1)\left(u_{i}^{0}(t)+\left(\delta_{i}(t)-1\right) \tilde{\alpha}_{i}(t)+\eta_{i}(t) \tilde{\beta}_{i}(t)\right)-\gamma_{i}(t)\left(u_{i}^{0}(t)+\delta_{i}(t) \tilde{\alpha}_{i}(t)+\eta_{i}(t) \tilde{\beta}_{i}(t)\right)^{2} \\
& +\left[p_{i}^{0}(t)+\theta_{i}(t) \tilde{\alpha}_{i}(t)+\rho_{i}(t) \tilde{\beta}_{i}(t)\right]\left[\tilde{\alpha}_{i}(t)-\tilde{\beta}_{i}(t)\left(p_{i}^{0}(t)+\theta_{i}(t) \tilde{\alpha}_{i}(t)+\rho_{i}(t) \tilde{\beta}_{i}(t)+H_{i}(t+1)\right)\right] \\
& M_{i}(t, X)=h_{i}(t+1) I_{i}^{0}+\min _{y, z} m_{i}(t, X, y, z) \\
& \text { s.t. }-1 \leq y, z \leq 1, \quad|y|+|z| \leq \Gamma_{i}(t) \\
& \tilde{\alpha}_{i}(t)=\alpha_{i}(t)+z \hat{\alpha}_{i}(t), \quad \tilde{\beta}_{i}(t)=\beta_{i}(t)+y \hat{\beta}_{i}(t)
\end{aligned}
$$

Proof. To prove the theorem, we consider decision variables $X$ fixed. We have to determine for each constraint (given for some product $i$ and time $t$ ) the realization of uncertain parameters $\left(\tilde{\alpha}_{i}(t), \tilde{\beta}_{i}(t)\right)$ that is least favorable to satisfying the constraint. Precisely, suppose a constraint consists of an expression involving uncertain parameters subject to a fixed upper bound. Then the robust counterpart for this constraint is obtained by guaranteeing that the maximum of the expression (over all feasible realizations of the uncertain parameters) satisfies the upper bound. Note that the robust counterpart constraint no longer involves any uncertain data. Moreover, it ensures that for any feasible realization, the upper bound will be satisfied.

We start with the constraints that are linear in the uncertain parameters: (4), (6) and (7). We then address constraints (5) and (8) that are quadratic in the uncertain parameters. Finally, we focus on constraint (3) that is a polynomial of degree 3 in the uncertain parameters.

Since $\mathcal{F}$ is separable across time and across products, the deterministic counterpart of constraint (4) is

$$
\begin{aligned}
& \sum_{i=1}^{N}\left(u_{i}^{0}(t)+\delta_{i}(t) \alpha_{i}(t)+\eta_{i}(t) \beta_{i}(t)+\max _{-1 \leq y, z \leq 1:|y|+|z| \leq \Gamma_{i}(t)} \delta_{i}(t) z \hat{\alpha}_{i}(t)+\eta_{i}(t) y \hat{\beta}_{i}(t)\right) \leq K(t) \\
\Leftrightarrow \quad & \sum_{i=1}^{N}\left(u_{i}^{0}(t)+\delta_{i}(t) \alpha_{i}(t)+\eta_{i}(t) \beta_{i}(t)+A_{i}\left(t, \delta_{i}(t), \eta_{i}(t)\right)\right) \leq K(t),
\end{aligned}
$$

where

$$
\begin{aligned}
A_{i}\left(t, \delta_{i}(t), \eta_{i}(t)\right)= & \begin{cases}\left|\delta_{i}(t)\right| \hat{\alpha}_{i}(t)+\left|\eta_{i}(t)\right| \hat{\beta}_{i}(t), & \Gamma_{i}(t) \geq 2 \\
\max \left\{\left|\delta_{i}(t)\right| \hat{\alpha}_{i}(t) ;\left|\eta_{i}(t)\right| \hat{\beta}_{i}(t)\right\}+\left(\Gamma_{i}(t)-1\right) \min \left\{\left|\delta_{i}(t)\right| \hat{\alpha}_{i}(t) ;\left|\eta_{i}(t)\right| \hat{\beta}_{i}(t)\right\}, & 1 \leq \Gamma_{i}(t)< \\
\Gamma_{i}(t) \max \left\{\left|\delta_{i}(t)\right| \hat{\alpha}_{i}(t) ;\left|\eta_{i}(t)\right| \hat{\beta}_{i}(t)\right\}, & 0 \leq \Gamma_{i}(t)<\end{cases} \\
=\min \left\{\Gamma_{i}(t) ; 1\right\} \max \left\{\left|\delta_{i}(t)\right| \hat{\alpha}_{i}(t) ;\left|\eta_{i}(t)\right| \hat{\beta}_{i}(t)\right\} & \\
& \quad+\min \left\{\left(\Gamma_{i}(t)-1\right)^{+} ; 1\right\} \min \left\{\left|\delta_{i}(t)\right| \hat{\alpha}_{i}(t) ;\left|\eta_{i}(t)\right| \hat{\beta}_{i}(t)\right\}
\end{aligned}
$$

Similarly, the deterministic counterpart of constraints (6) and (7) are respectively

$$
\begin{aligned}
(6) & \Leftrightarrow u_{i}^{0}(t)+\delta_{i}(t) \alpha_{i}(t)+\eta_{i}(t) \beta_{i}(t)+\min _{-1 \leq y, z \leq 1:|y|+|z| \leq \Gamma_{i}(t)} \delta_{i}(t) z \hat{\alpha}_{i}(t)+\eta_{i}(t) y \hat{\beta}_{i}(t) \geq 0 \\
& \Leftrightarrow u_{i}^{0}(t)+\delta_{i}(t) \alpha_{i}(t)+\eta_{i}(t) \beta_{i}(t)-A_{i}\left(t, \delta_{i}(t), \eta_{i}(t)\right) \geq 0 \\
(7) & \Leftrightarrow p_{i}^{0}(t)+\theta_{i}(t) \alpha_{i}(t)+\rho_{i}(t) \beta_{i}(t)+\min _{-1 \leq y, z \leq 1:|y|+|z| \leq \Gamma_{i}(t)} \theta_{i}(t) z \hat{\alpha}_{i}(t)+\rho_{i}(t) y \hat{\beta}_{i}(t) \geq 0 \\
& \Leftrightarrow p_{i}^{0}(t)+\theta_{i}(t) \alpha_{i}(t)+\rho_{i}(t) \beta_{i}(t)-A_{i}\left(t, \theta_{i}(t), \rho_{i}(t)\right) \geq 0,
\end{aligned}
$$


We observe that the deterministic counterpart of constraint (8) is

$$
\begin{gathered}
B_{i}(t, X) \equiv \quad I_{i}^{0}+\sum_{s=0}^{t-1}\left(u_{i}^{0}(s)+\delta_{i}(s) \alpha_{i}(s)+\eta_{i}(s) \beta_{i}(s)\right)-\sum_{s=0}^{t-1}\left(\alpha_{i}(s)-\beta_{i}(s)\left(p_{i}^{0}(s)+\theta_{i}(s) \alpha_{i}(s)+\rho_{i}(s) \beta_{i}(s)\right)\right. \\
+\sum_{s=0}^{t-1} \min _{-1 \leq y, z \leq 1:|y|+|z| \leq \Gamma_{i}(s)}\left(z \hat{\alpha}_{i}(s)\left(\delta_{i}(s)-1+\beta_{i}(s) \theta_{i}(s)\right)\right. \\
+y \hat{\beta}_{i}(s)\left(\eta_{i}(s)+p_{i}^{0}(s)+\theta_{i}(s) \alpha_{i}(s)+2 \rho_{i}(s) \beta_{i}(s)\right) \\
\left.+y^{2} \hat{\beta}_{i}(s)^{2} \rho_{i}(s)+y z \theta_{i}(s) \hat{\beta}_{i}(s) \hat{\alpha}_{i}(s)\right) \geq 0
\end{gathered}
$$

We notice that the subproblem involved above is no longer linear as for the previous constraints, but quadratic, with linear constraints. We do not provide the closed-form solution, but such a subproblem is easy to solve.

Similarly, obtaining the deterministic counterpart of constraint (5) involves solving a quadratic program with linear constraints:

$$
\begin{aligned}
(5) \Leftrightarrow & C_{i}(t, X) \equiv \beta_{i}(t)\left(p_{i}^{0}(t)+\theta_{i}(t) \alpha_{i}(t)+\rho_{i}(t) \beta_{i}(t)\right)-\alpha_{i}(t)+\max _{-1 \leq y, z \leq 1:|y|+|z| \leq \Gamma_{i}(t)}\left(z \hat{\alpha}_{i}(t)\left(\beta_{i}(t) \theta_{i}(t)-1\right)\right. \\
& \left.+y \hat{\beta}_{i}(t)\left(p_{i}^{0}(t)+\theta_{i}(t) \alpha_{i}(t)+2 \rho_{i}(t) \beta_{i}(t)\right)+y^{2} \hat{\beta}_{i}(t)^{2} \rho_{i}(t)+y z \theta_{i}(t) \hat{\beta}_{i}(t) \hat{\alpha}_{i}(t)\right) \leq 0
\end{aligned}
$$

A similar reasoning leads to proving that the deterministic counterpart of constraint (3) involves a subproblem with an objective that is a polynomial of degree 3 , and linear constraints. We show this below.

Since

$$
\sum_{t=0}^{T-1} h_{i}(t+1) \tilde{I}_{i}(t+1)=\sum_{t=0}^{T-1} h_{i}(t+1) I_{i}^{0}+H_{i}(t+1)\left(u_{i}(t)-\tilde{\alpha}_{i}(t)+\tilde{\beta}_{i}(t) p_{i}(t)\right),
$$

where $H_{i}(t)$ was defined in Section 4.1 as $H_{i}(t)=\sum_{t=s}^{T} h_{i}(s)$, we have

$$
\begin{aligned}
(3) \Leftrightarrow Q \leq & \sum_{i=1}^{N} \sum_{t=0}^{T-1} h_{i}(t+1) I_{i}^{0}-H_{i}(t+1)\left(u_{i}^{0}(t)+\delta_{i}(t) \tilde{\alpha}_{i}(t)+\eta_{i}(t) \tilde{\beta}_{i}(t)-\tilde{\alpha}_{i}(t)\right) \\
& +\left[p_{i}^{0}(t)+\theta_{i}(t) \tilde{\alpha}_{i}(t)+\rho_{i}(t) \tilde{\beta}_{i}(t)\right]\left[\tilde{\alpha}_{i}(t)-\tilde{\beta}_{i}(t)\left(p_{i}^{0}(t)+\theta_{i}(t) \tilde{\alpha}_{i}(t)+\rho_{i}(t) \tilde{\beta}_{i}(t)+H_{i}(t+1)\right)\right] \\
& -\gamma_{i}(t)\left(u_{i}^{0}(t)+\delta_{i}(t) \tilde{\alpha}_{i}(t)+\eta_{i}(t) \tilde{\beta}_{i}(t)\right)^{2} \quad \forall(\tilde{\alpha}(t), \tilde{\beta}(t)) \in \mathcal{F}(t)
\end{aligned}
$$

To obtain the counterpart, we need to minimize, for fixed $i$ and $t$, the addend in the right-hand side above, over $y$ and $z$ such that $-1 \leq y, z \leq 1, \quad|y|+|z| \leq \Gamma_{i}(t)$, where $\tilde{\alpha}_{i}(t)=\alpha_{i}(t)+z \hat{\alpha}_{i}(t), \quad \tilde{\beta}_{i}(t)=$ $\beta_{i}(t)+y \hat{\beta}_{i}(t)$. The addend being of degree 3 in $\tilde{\alpha}_{i}(t)$ and $\tilde{\beta}_{i}(t)$, it is of degree 3 in $y$ and $z$.

We thus prove the theorem.

Note that since the solutions of the subproblems involved in this formulation depend on vector $X$, the resulting problem has a nonlinear objective and nonlinear constraints. 


\section{Open-loop Problem}

In this section, we present open-loop formulation via both robust optimization and stochastic optimization, mainly for comparison purposes with closed-loop formulations. An open-loop model is justified if the firm must make an irreversible commitment to a future course of action. This situation may arise in practice if a contract with another firm or with a labor union forces the firm to commit to prices or workforce at the beginning of the time horizon. However, in many applications firms may review their course of action as time evolves, depending on the actual realization of uncertain data so far. Therefore in most cases closed-loop models are more reasonable than openloop models, which is why we primarily introduced closed-loop formulations in Section 3. In this section, we derive open-loop solutions to compare the complexity, probability of constraint violation, and profits with closed-loop solutions.

\subsection{Robust Optimization}

\subsubsection{Formulation}

We assume that budgets of uncertainty are taken across uncertain variables, for a given product and a given time. Uncertainty set $\mathcal{F}$ can then be viewed as $\mathcal{F}=\prod_{i=1}^{N} \prod_{t=0}^{T-1} \mathcal{F}_{i}(t)$, where

$$
\mathcal{F}_{i}(t)=\left\{\left(\tilde{\alpha}_{i}(t), \tilde{\beta}_{i}(t)\right):\left|\frac{\tilde{\alpha}_{i}(t)-\alpha_{i}(t)}{\hat{\alpha}_{i}(t)}\right|+\left|\frac{\tilde{\beta}_{i}(t)-\beta_{i}(t)}{\hat{\beta}_{i}(t)}\right| \leq \Gamma_{i}(t)\right\}
$$

The problem is formulated as follows:

$$
\begin{array}{cl}
\underset{u(.), p(.)}{\max } & Q \\
\text { s.t. } & Q \leq \sum_{i=1}^{N} \sum_{t=0}^{T-1} p_{i}(t)\left(\tilde{\alpha}_{i}(t)-\tilde{\beta}_{i}(t) p_{i}(t)\right)-\gamma_{i}(t) u_{i}(t)^{2} \\
& \quad-h_{i}(t+1) \tilde{I}_{i}(t+1) \quad \forall(\tilde{\alpha}, \tilde{\beta}) \in \mathcal{F} \\
& \tilde{I}_{i}(t+1)=\tilde{I}_{i}(t)+u_{i}(t)-\left(\tilde{\alpha}_{i}(t)-\tilde{\beta}_{i}(t) p_{i}(t)\right), \quad \tilde{I}_{i}(0)=I_{i}^{0} \quad \forall i, t \\
& \sum_{i=1}^{N} u_{i}(t) \leq K(t) \quad \forall t \\
& \tilde{I}_{i}(t) \geq 0 \quad \forall i, t \quad \forall(\tilde{\alpha}, \tilde{\beta}) \in \mathcal{F} \\
& p_{i}(t) \leq \frac{\tilde{\alpha}_{i}(t)}{\tilde{\beta}_{i}(t)} \quad \forall i, t \quad \forall(\tilde{\alpha}, \tilde{\beta}) \in \mathcal{F} \\
& u_{i}(t), \quad p_{i}(t) \geq 0 \quad \forall i, t
\end{array}
$$

\subsubsection{Robust counterpart problem}

To rewrite deterministically the upper bound on prices (15) in the robust counterpart, we must solve, for each fixed $i$ and $t$,

$$
\min _{\left(\tilde{\alpha}_{i}(t), \tilde{\beta}_{i}(t)\right) \in \mathcal{F}_{i}(t)} \frac{\tilde{\alpha}_{i}(t)}{\tilde{\beta}_{i}(t)}
$$


We can show that the solution of this optimization problem is

$$
p_{i}^{\max }(t)=\min _{y, z \in[-1,1]:|y|+|z| \leq \Gamma_{i}(t)} \frac{\alpha_{i}(t)+z \hat{\alpha}_{i}(t)}{\beta_{i}(t)+y \hat{\beta}_{i}(t)}= \begin{cases}\frac{\alpha_{i}(t)}{\beta_{i}(t)+\Gamma_{i}(t) \hat{\beta}_{i}(t)}, & \Gamma_{i}(t) \leq \min \left\{1, \frac{\alpha_{i}(t)}{\hat{\alpha}_{i}(t)}-\frac{\beta_{i}(t)}{\hat{\beta}_{i}(t)}\right\} \\ \frac{\alpha_{i}(t)-\left(\Gamma_{i}(t)-1\right) \hat{\alpha}_{i}(t)}{\beta_{i}(t)+\hat{\beta}_{i}(t)}, & 1<\Gamma_{i}(t) \leq \min \left\{2, \frac{\alpha_{i}(t)}{\hat{\alpha}_{i}(t)}-\frac{\beta_{i}(t)}{\hat{\beta}_{i}(t)}\right\} \\ \frac{\alpha_{i}(t)-\Gamma_{i}(t) \hat{\alpha}_{i}(t)}{\beta_{i}(t)}, & \frac{\alpha_{i}(t)}{\hat{\alpha}_{i}(t)}-\frac{\beta_{i}(t)}{\hat{\beta}_{i}(t)}<\Gamma_{i}(t) \leq 1 \\ \frac{\alpha_{i}(t)-\hat{\alpha}_{i}(t)}{\beta_{i}(t)+\left(\Gamma_{i}(t)-1\right) \hat{\beta}_{i}(t)}, & \max \left\{\frac{\alpha_{i}(t)}{\hat{\alpha}_{i}(t)}-\frac{\beta_{i}(t)}{\hat{\beta}_{i}(t)}, 1\right\}<\Gamma_{i}(t) \leq 2 \\ \frac{\alpha_{i}(t)-\hat{\alpha}_{i}(t)}{\beta_{i}(t)+\hat{\beta}_{i}(t)}, & \Gamma_{i}(t)>2\end{cases}
$$

As the no stockout constraint (14) is least favored by low realized inventory levels, i.e. high demand, constraint (14) can be rewritten by solving, for fixed $p_{i}($.$) and for s=0 \ldots, t-1$,

$$
\max _{\left(\tilde{\alpha}_{i}(s), \tilde{\beta}_{i}(s)\right) \in \mathcal{F}_{i}(s)} \tilde{\alpha}_{i}(s)-\tilde{\beta}_{i}(s) p_{i}(s)
$$

which we can show equals

$\alpha_{i}(s)-\beta_{i}(s) p_{i}(s)+\min \left\{1, \Gamma_{i}(s)\right\} \max \left\{\hat{\alpha}_{i}(s), \hat{\beta}_{i}(s) p_{i}(s)\right\}+\min \left\{1,\left(\Gamma_{i}(s)-1\right)^{+}\right\} \min \left\{\hat{\alpha}_{i}(s), \hat{\beta}_{i}(s) p_{i}(s)\right\}$.

In (13), the worst realized profits incurred at time period $t$ correspond to low revenue and high inventories at the end of the time period, i.e. low demand from time 0 to time $t$. Thus we can find the most adverse realization separately for the revenue term and for the holding cost term, and the counterpart involves solving, for each $i$ and $t$,

$$
\min _{\left(\tilde{\alpha}_{i}(t), \tilde{\beta}_{i}(t)\right) \in \mathcal{F}_{i}(t)} \tilde{\alpha}_{i}(t)-\tilde{\beta}_{i}(t) p_{i}(t)
$$

which we can show equals

$\alpha_{i}(t)-\beta_{i}(t) p_{i}(t)-\min \left\{1, \Gamma_{i}(t)\right\} \max \left\{\hat{\alpha}_{i}(t), \hat{\beta}_{i}(t) p_{i}(t)\right\}-\min \left\{1,\left(\Gamma_{i}(t)-1\right)^{+}\right\} \min \left\{\hat{\alpha}_{i}(t), \hat{\beta}_{i}(t) p_{i}(t)\right\}$.

In particular we obtain

$$
\begin{aligned}
& \max _{(\tilde{\alpha}, \tilde{\beta}) \in \mathcal{F}} \sum_{t=0}^{T-1} h_{i}(t+1) \tilde{I}_{i}(t+1)=\sum_{t=0}^{T-1} h_{i}(t+1) I_{i}(t+1)+ \\
& \sum_{t=1}^{T} H_{i}(t)\left(\min \left\{1, \Gamma_{i}(t)\right\} \max \left\{\hat{\alpha}_{i}(t), \hat{\beta}_{i}(t) p_{i}(t)\right\}+\min \left\{1,\left(\Gamma_{i}(t)-1\right)^{+}\right\} \min \left\{\hat{\alpha}_{i}(t), \hat{\beta}_{i}(t) p_{i}(t)\right\}\right)
\end{aligned}
$$

where $H_{i}(t)=\sum_{s=t}^{T} h_{i}(s)$ and $I_{i}(t+1)$ is the nominal inventory level.

Therefore the robust counterpart problem is written as a convex quadratic program with linear constraints, where the no stockout constraint is replaced with a minimum inventory security level, 
and the upper bound on prices is tighter than in the nominal problem:

$$
\begin{aligned}
\max _{u(.), p(.)} \quad & \sum_{i=1}^{N} \sum_{t=0}^{T-1}\left(p_{i}(t)\left(\alpha_{i}(t)-\beta_{i}(t) p_{i}(t)\right)-q_{i}(t+1)\left(p_{i}(t+1)+H_{i}(t+1)\right) \min \left\{1, \Gamma_{i}(t+1)\right\}\right. \\
& \left.-r_{i}(t+1)\left(p_{i}(t+1)+H_{i}(t+1)\right) \min \left\{1,\left(\Gamma_{i}(t+1)-1\right)^{+}\right\}-\gamma_{i}(t) u_{i}(t)^{2}-h_{i}(t+1) I_{i}(t+1)\right) \\
& q_{i}(t) \geq \hat{\alpha}_{i}(t) \geq r_{i}(t) \quad \forall i, t \\
& q_{i}(t) \geq \hat{\beta}_{i}(t) p_{i}(t) \geq r_{i}(t) \quad \forall i, t \\
& I_{i}(t+1) \equiv I_{i}(t)+u_{i}(t)-\left(\alpha_{i}(t)-\beta_{i}(t) p_{i}(t)\right), \quad I_{i}(0)=I_{i}^{0} \quad \forall i, t \\
& \sum_{i=1}^{N} u_{i}(t) \leq K(t) \forall t \\
& I_{i}(t) \geq \sum_{s=1}^{t} \min \left\{1, \Gamma_{i}(s)\right\} q_{i}(s)+\min \left\{1,\left(\Gamma_{i}(s)-1\right)^{+}\right\} r_{i}(s) \forall i, t \\
& p_{i}(t) \leq p_{i}^{\max }(t) \forall i, t \\
& u_{i}(t), \quad p_{i}(t) \geq 0 \quad \forall i, t
\end{aligned}
$$

where $H_{i}(t)=\sum_{s=t}^{T} h_{i}(s)$. Therefore, the robust counterpart is of the same order of complexity as the nominal problem.

\subsection{Stochastic Optimization}

When we assume that the probability distributions of the demand parameters are known, we solve:

$$
\begin{aligned}
\max _{p_{i}(t), u_{i}(t), \forall i, t} & \sum_{i=1}^{N} \sum_{t=0}^{T-1} E\left[p_{i}(t)\left(\tilde{\alpha}_{i}(t)-\tilde{\beta}_{i}(t) p_{i}(t)\right)-\gamma_{i}(t) u_{i}(t)^{2}-h_{i}(t+1) \tilde{I}_{i}(t+1)\right] \\
\text { s.t. } \quad & \sum_{i=1}^{N} u_{i}(t) \leq K(t), \quad t=0, \ldots, T-1 \\
& P\left(\tilde{I}_{i}(t) \geq 0\right) \geq 1-\epsilon, \quad t=1, \ldots, T, \quad i=1, \ldots, N \\
& P\left(p_{i}(t) \leq \frac{\tilde{\alpha}_{i}(t)}{\tilde{\beta}_{i}(t)} \geq 1-\epsilon, \quad t=0, \ldots, T-1, \quad i=1, \ldots, N\right. \\
& u_{i}(t), p_{i}(t) \geq 0, \quad t=0, \ldots, T-1, \quad i=1, \ldots, N \\
& \tilde{I}_{i}(t) \equiv I_{i}^{0}+\sum_{s=0}^{t-1} u_{i}(s)-\sum_{s=0}^{t-1}\left(\tilde{\alpha}_{i}(s)-\tilde{\beta}_{i}(s) p_{i}(s)\right), \quad t=1, \ldots, T, \quad i=1, \ldots, N .
\end{aligned}
$$

Note that the expectation above is taken over $\tilde{\alpha}_{i}(),. \tilde{\beta}_{i}(),. i=1, \ldots, N$. Notice that, by linearity in the uncertain data of the inventory level and profits, the expected profits equal the nominal profits. The complexity of solving this problem depends essentially on the structure of the chance constraints (16) and (17). In particular, for this problem, they involve the convolution of distributions of uncertain parameters. Therefore, they become more complex if $\tilde{\alpha}_{i}(t)$ and $\tilde{\beta}_{i}(t)$ follow different types of distribution or if the probability distribution is not stable (i.e. a linear combination does not necessarily follow the same type of distribution). 
In the case of Normal distributions (that are stable), let $\varphi($.$) be the cumulative distribution function$ of the standard Normal random variable. Then an equivalent formulation is:

$$
\begin{aligned}
\max _{p_{i}(t), u_{i}(t), \forall i, t} & \sum_{i=1}^{N} \sum_{t=0}^{T-1}\left(p_{i}(t)\left(\alpha_{i}(t)-\beta_{i}(t) p_{i}(t)\right)-\gamma_{i}(t) u_{i}(t)^{2}-h_{i}(t+1) I_{i}(t+1)\right) \\
\text { s.t. } \quad & \sum_{i=1}^{N} u_{i}(t) \leq K(t), \quad t=0, \ldots, T-1 \\
& I_{i}(t) \geq 0, \quad t=1, \ldots, T, \quad i=1, \ldots, N \\
& 4 I_{i}(t)^{2} \geq\left(\varphi^{-1}(\epsilon)\right)^{2} \sum_{s=1}^{t}\left(\hat{\alpha}_{i}(s)^{2}+p_{i}(s)^{2} \hat{\beta}_{i}(s)^{2}\right), \quad t=1, \ldots, T, \quad i=1, \ldots, N \\
& p_{i}(t) \leq \frac{\alpha_{i}(t)}{\beta_{i}(t)}, \quad t=0, \ldots, T-1, \quad i=1, \ldots, N \\
& 4\left(\alpha_{i}(t)-\beta_{i}(t) p_{i}(t)\right)^{2} \geq\left(\varphi^{-1}(\epsilon)\right)^{2}\left(\hat{\alpha}_{i}(t)^{2}+p_{i}(t)^{2} \hat{\beta}_{i}(t)^{2}\right) \quad t=0, \ldots, T-1, \quad i=1, \ldots, N \\
& u_{i}(t), p_{i}(t) \geq 0, \quad t=0, \ldots, T-1, \quad i=1, \ldots, N \\
& I_{i}(t) \equiv I_{i}^{0}+\sum_{s=1}^{t} u_{i}(s)-\left(\alpha_{i}(s)-\beta_{i}(s) p_{i}(s)\right), \quad t=1, \ldots, T, \quad i=1, \ldots, N .
\end{aligned}
$$

We notice that for the case when uncertain data are Normally distributed, the resulting formulation is a quadratic program with conic constraints, which is more complex than the nominal problem since constraints are now nonlinear. For other distributional assumptions, the constraints may not be quadratic, they would be nonlinear, with a structure that depends on the distribution. In particular, convexity is not guaranteed.

\section{$5 \quad$ Numerical Study}

We consider the 2-period, one product case introduced in Section 3.1 and further studied in Section 3.2. We implement the deterministic approach, perfect hindsight as a tool of comparison. We also implement the stochastic models (open-loop with chance constraints and dynamic programming) under the assumption that realizations follow on the one hand a Normal distribution, and on the other hand a uniform distribution. Furthermore, we solve the robust open-loop, adjustable robust, and affine adjustable models with budgets of uncertainty shared by parameters, and we consider a range of possible budgets. We generate random realizations based on a uniform distribution, in order to observe the effect of having made the right or wrong assumption on the actual distribution.

In open-loop, we will compare the probability of violating the constraints involving uncertainty in the robust and stochastic approach. We compute it as the number of times that the inventory level at the end of the time horizon is negative, out of the 1,000 randomly generated cases of demand parameters. Moreover, for each violation, we calculate the average amplitude of violation, i.e. the (negative) value reached by the inventory level.

In closed-loop, we will compare the performance of the dynamic program vs. adjustable and affine adjustable robust model, based on different budgets of uncertainty and distributional assumptions. We show below plots of the production rates, prices, inventory levels at both periods, overall realized profits, and in open-loop, probability and amplitude of violation. Note that when the value in 
consideration depends on the realization of the uncertain parameter (e.g. final inventory level), we plot the average over all 1,000 generated scenarios. We implement on the one hand for a range of production capacities (Figures 1, 3, and 5), and on the other hand for a range of budgets of uncertainty (Figures 2, 4, and 6). Notice that only the robust, adjustable robust, and affine adjustable robust solutions vary with the budget of uncertainty.

The inputs we choose are the following:

$$
\begin{array}{cll}
\alpha(0)=\alpha(1)=\alpha=15 & \beta(0)=\beta(1)=\beta=2 & h(1)=h(2)=h=0.8 \\
\gamma(0)=\gamma(1)=\gamma=2 & K(0)=K(1)=K & I^{0}=8, \quad \epsilon=5 \% \\
\hat{\alpha}(0)=\hat{\alpha}(1)=\hat{\alpha}=0.1 \alpha & \hat{\beta}(0)=\hat{\beta}(1)=\hat{\beta}=0.1 \beta & \\
\Gamma(1)=0.5,1, \quad 1.5, \quad 0<K \leq 1 \text { and } 0.7<\Gamma(1) \leq 1.6, \quad K=0.7 .
\end{array}
$$

We observe that as the budget increases, so does the inventory protection level, and thus so does the final inventory level. In the stochastic open-loop model, the chance constraints also translate implicitly into an inventory security level. As noted earlier, in closed-loop models, the final inventory level equals zero.

In Figures 5 and 6 we can observe the realized profits. However, it is important to notice that no arbitrary penalty was introduced in the case of constraint violation. As a result, since inventory costs are linear, negative inventories increase profits. Therefore profits must be looked at jointly with the probability of constraint violation, and by how much constraints are violated, shown in Figures 3 and 4. In particular, the deterministic model achieves high realized profits, even higher than the perfect hindsight, only because it often yields large negative inventories. Similarly, it would seem intuitive that the stochastic model that makes the correct (uniform) assumption on the distribution performs better that the other model. However, it yields lower profits than the model that assumed a Normal distribution, simply because it is less likely to violate the no stockout constraint. To better illustrate the tradeoff between risk (the probability and amplitude of constraint violation) and return (average profits), we plotted in Figures 7 and 8 all the data points on a risk/return diagram, where the vertical axis corresponds to the "risk", i.e. the average amplitude of constraint violation multiplied by the probability of a violation, and the horizontal axis corresponds to the average realized profits. Note that in Figure 8, we let the budget of uncertainty vary and therefore there is only one data points for models that are independent of $\Gamma(1)$, such that the deterministic and the stochastic models.

As this is a 2-period model, the closed-loop models are very close to the perfect hindsight, regardless of the budget of uncertainty. We conjecture that they would not be as close for many time periods. The open loop robust model allows to control the probability of constraint violation by varying the budget of uncertainty. The stochastic model that had the correct assumption achieves a probability of violation of $\epsilon$ as we aimed for. However, if the wrong assumption was made, the chance of violation exceeds the tolerance significantly. Therefore, for a sensitive constraint, it is crucial in the stochastic model to be sure about the probability distribution, as otherwise it is difficult to predict the probability of constraint violation yielded by the solution. In this case, the robust model seems to be a good alternative.

We notice that the affinely adjustable robust solution performs very well compared with the robust 

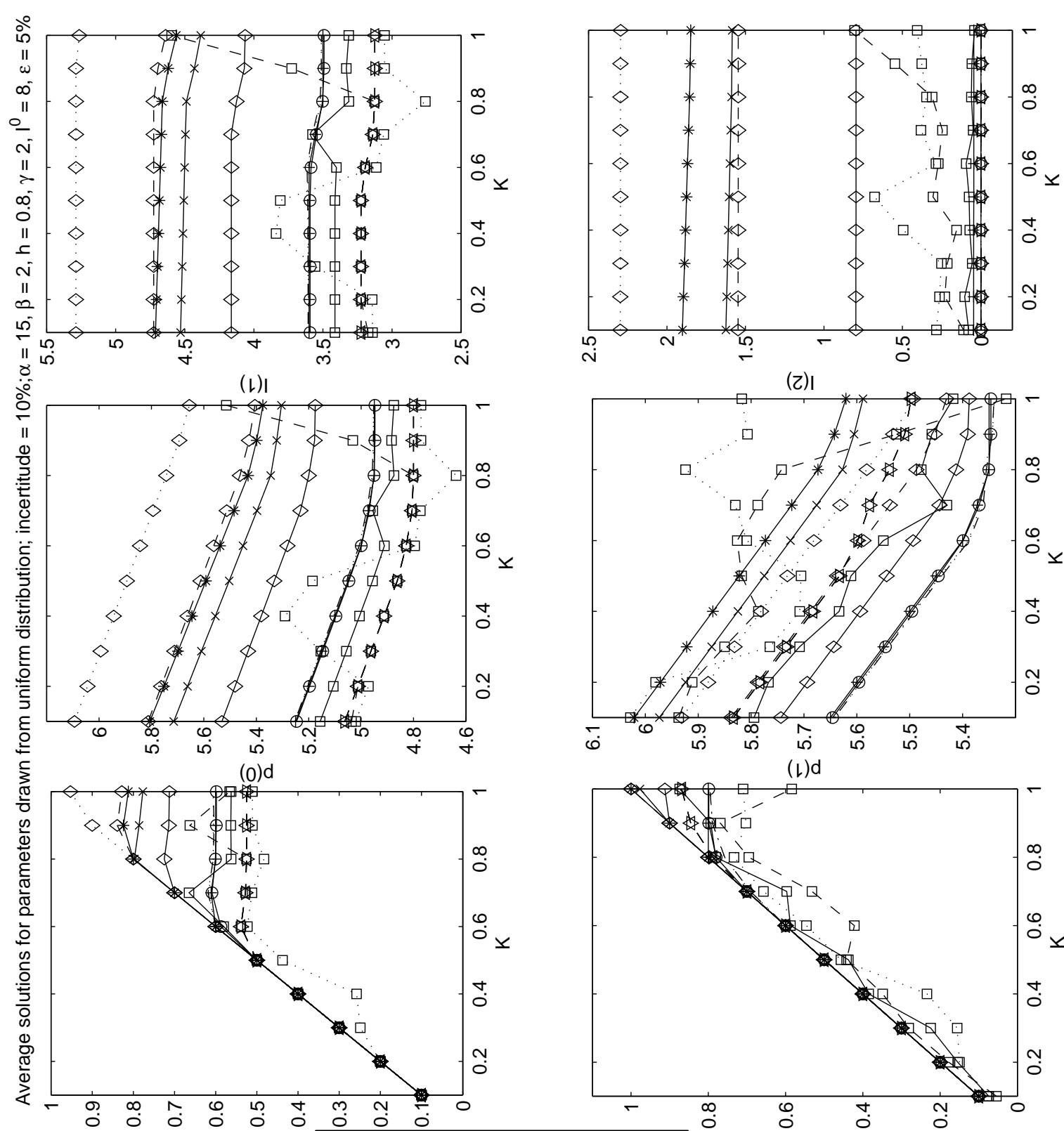

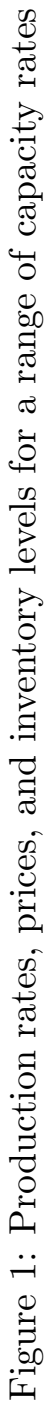

(0)n

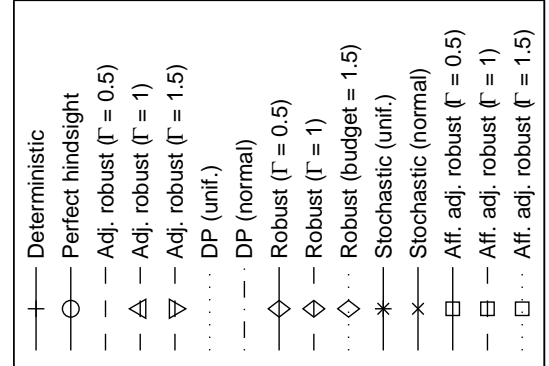

(1) $n$

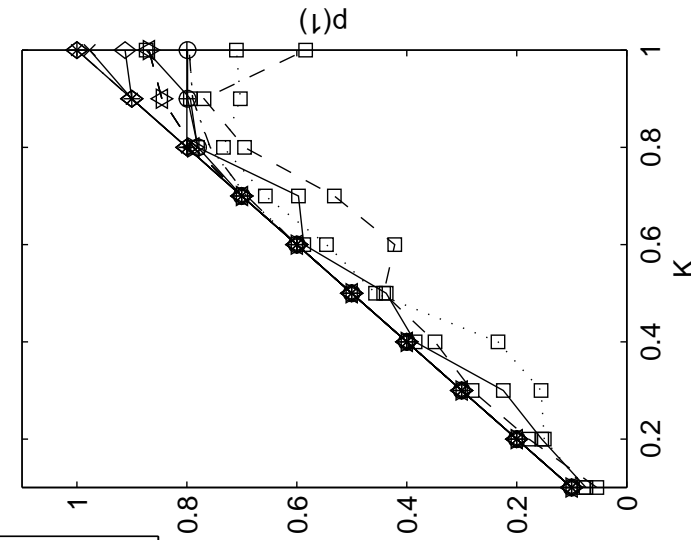




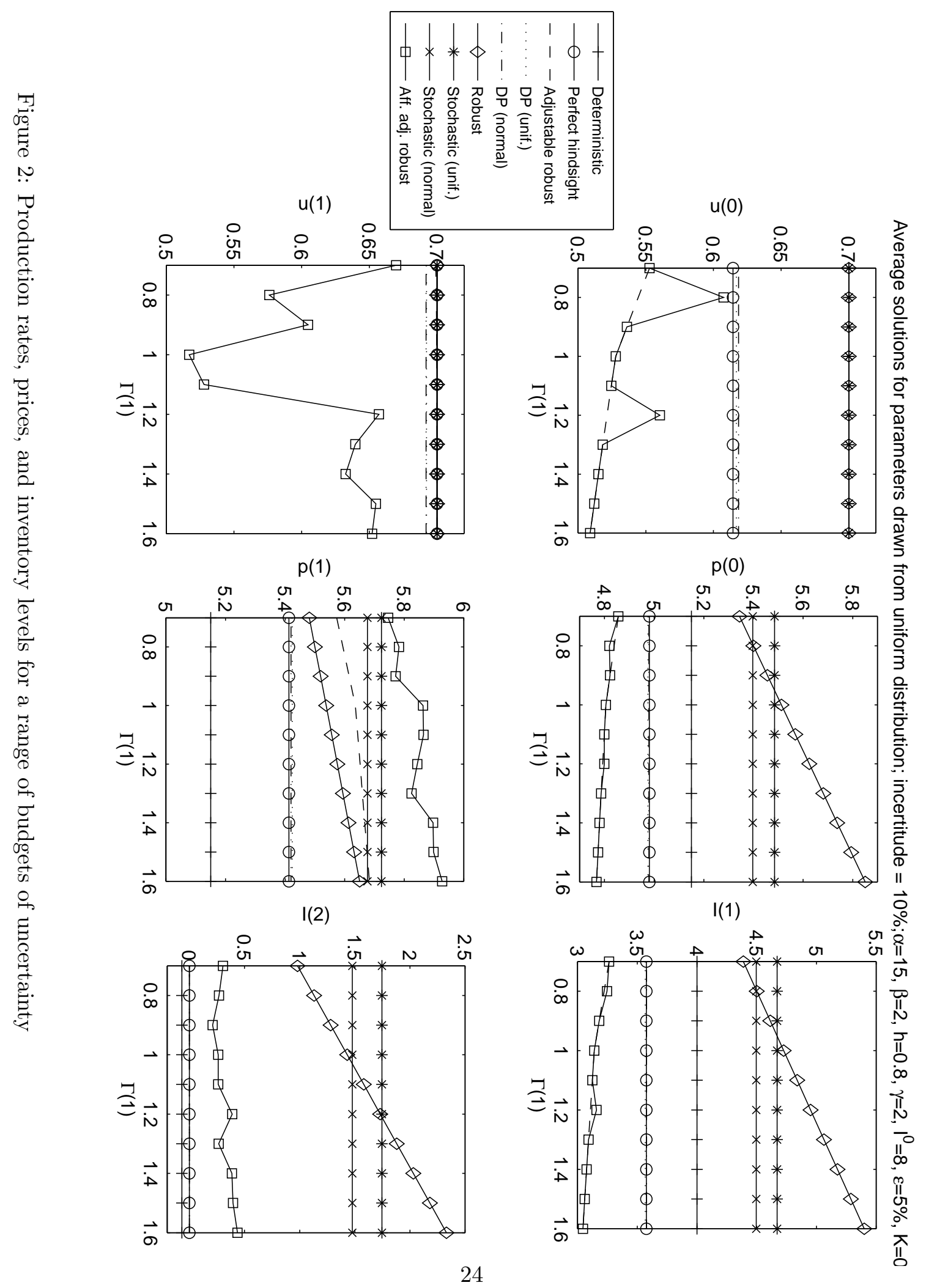



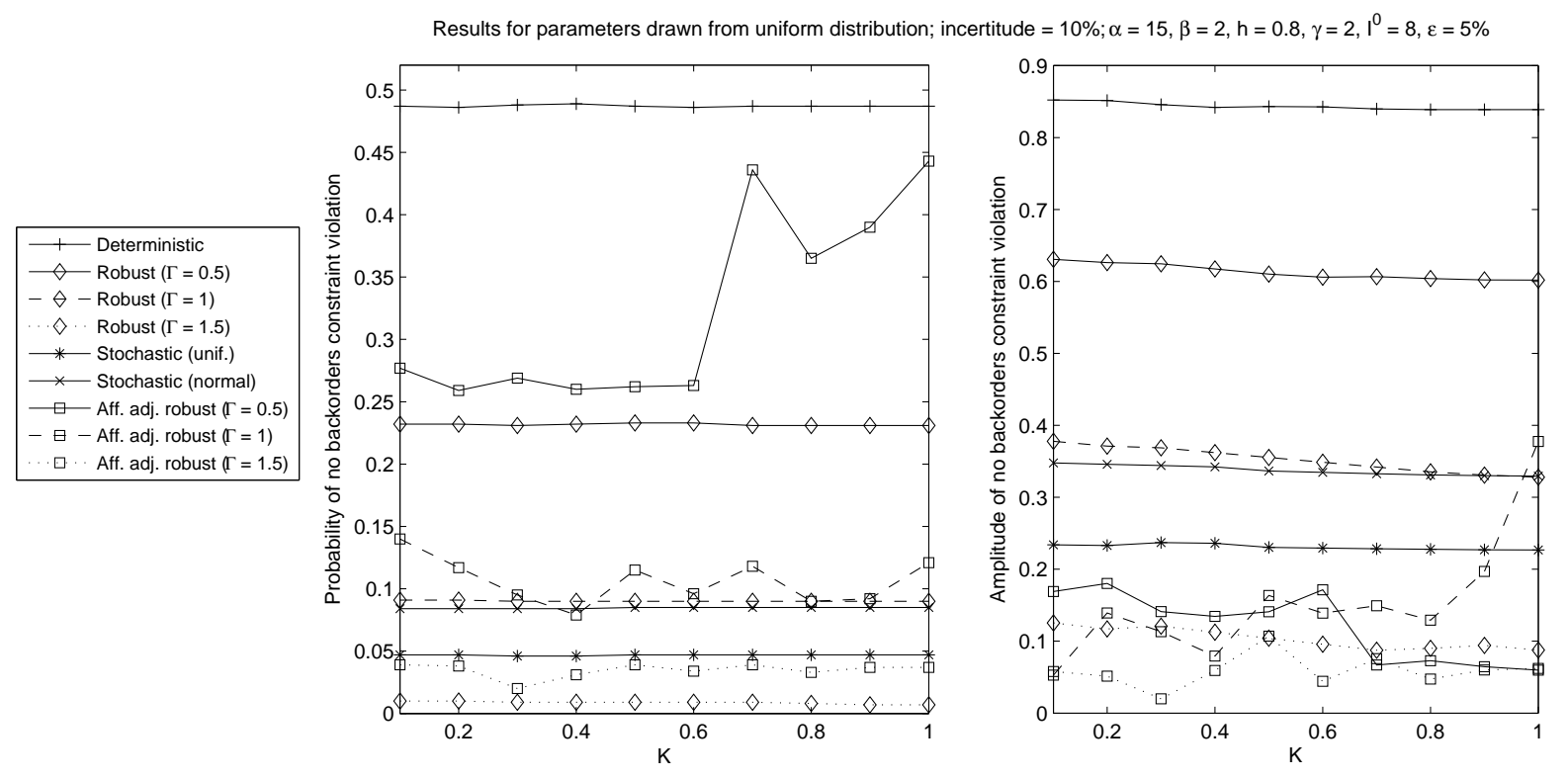

Figure 3: Probability and maximum amplitude of constraint violation for a range of capacity rates
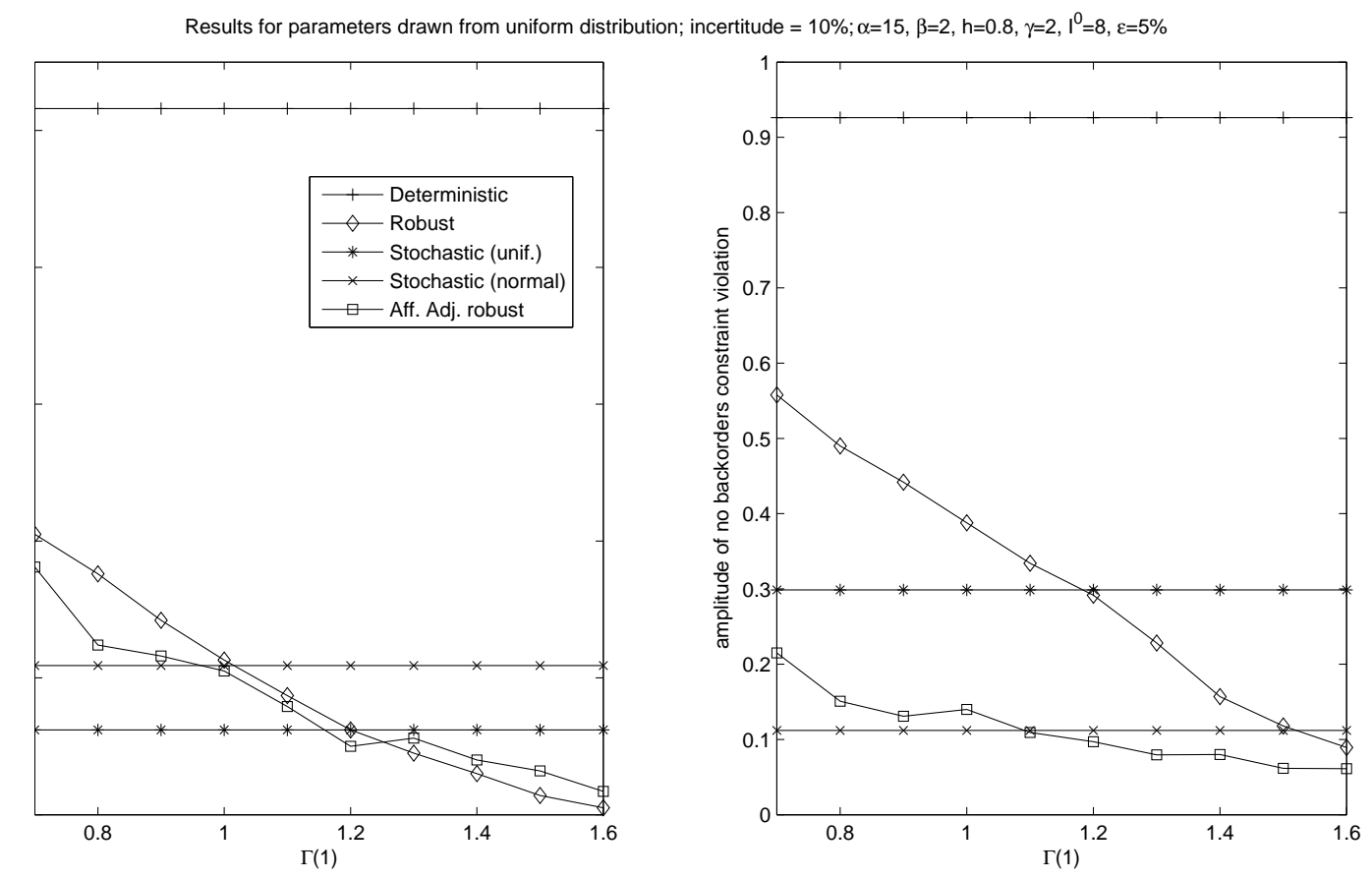

Figure 4: Probability and maximum amplitude of constraint violation for a range of budgets of uncertainty 


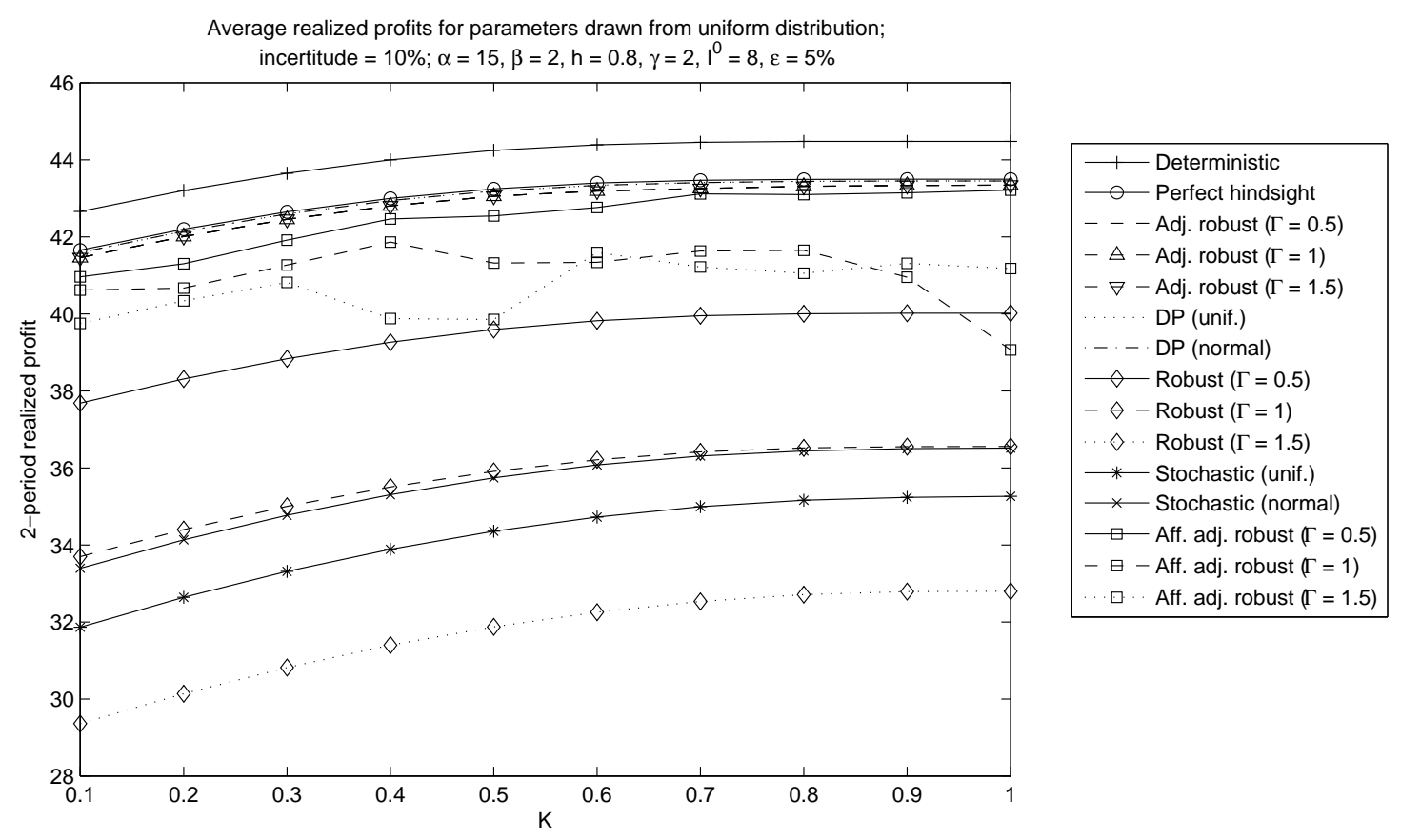

Figure 5: Profits for a range of capacity rates

Average realized profits for parameters drawn from uniform distribution; incertitude $=10 \% ; \alpha=15, \beta=2, h=0.8, \gamma=2, I^{0}=8, \varepsilon=5 \% ; K=0.7$

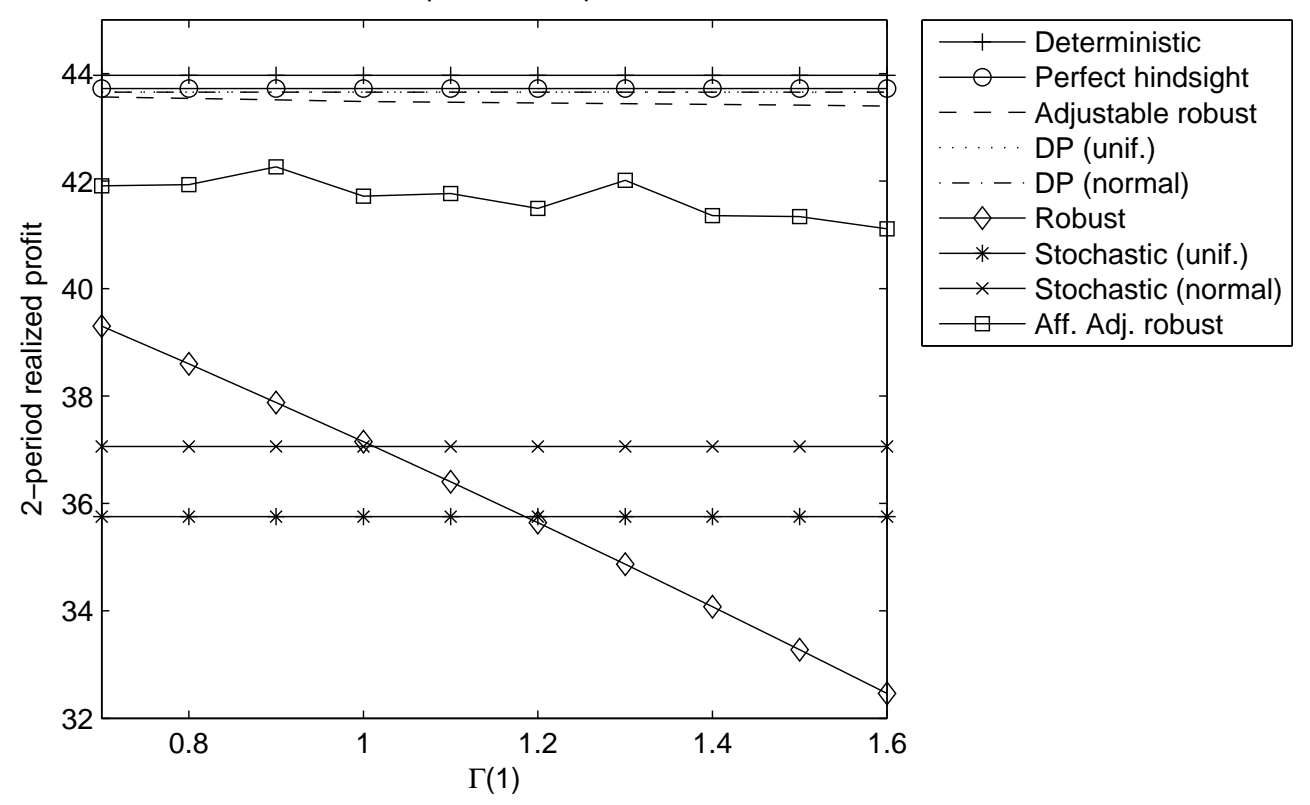

Figure 6: Profits for a range of budgets of uncertainty 


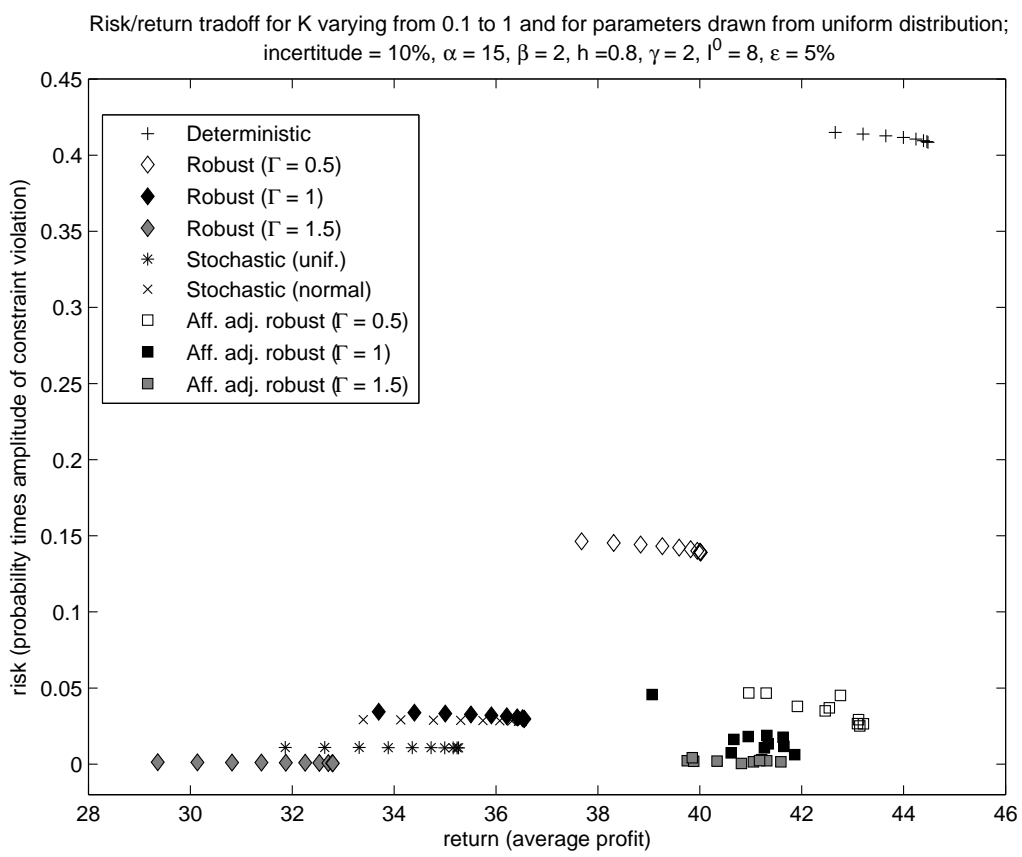

Figure 7: Risk return diagram for a range of capacity rates from 0.1 to 1

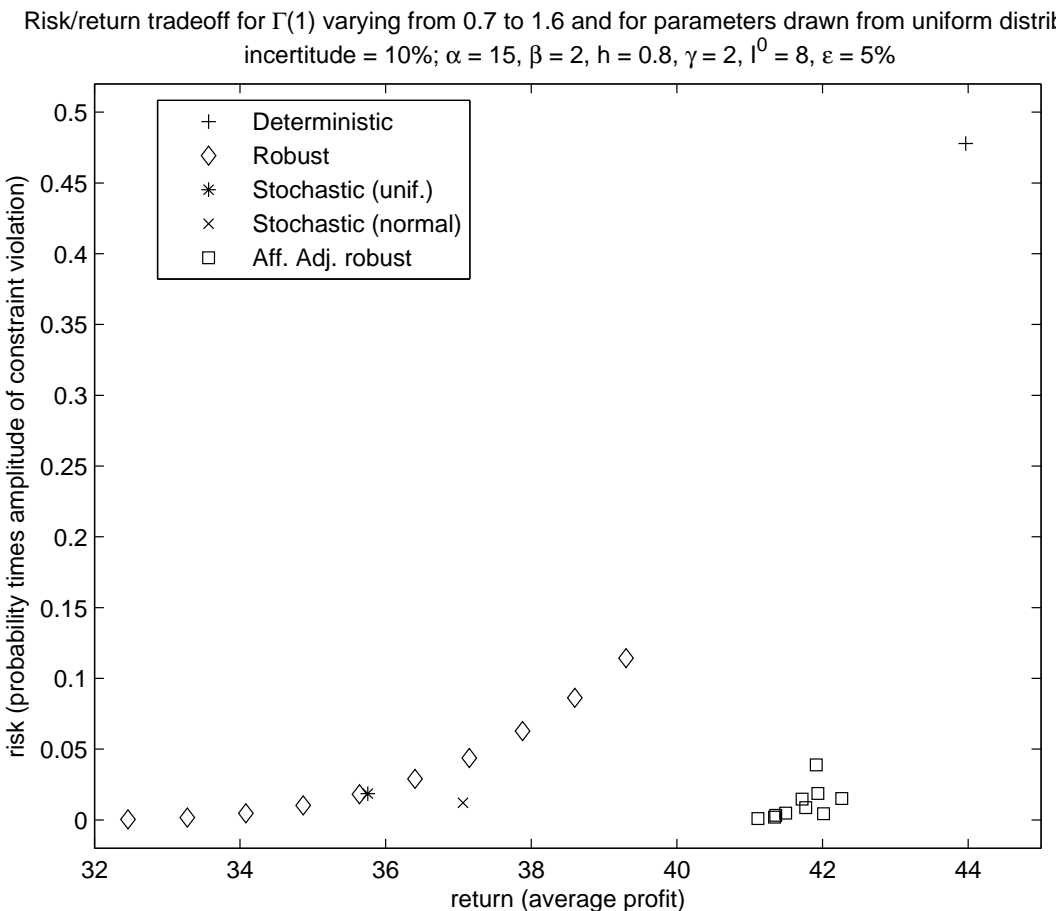

Figure 8: Risk return diagram for a range of budgets of uncertainty $\Gamma(1)$ from 0.7 to 1.6 
and stochastic formulations. All three models involve solving an optimization problem at time zero (even though in the affinely adjustable robust formulation, the actual price and production decisions are not calculated until after the demand realizes). The affinely adjustable robust solution avoids constraint violation and yields higher profits than the other open-loop models. As can be seen on the risk return diagrams, it yields high returns with low risk compared with other models. Moreover, the overall profits decrease as the budget of uncertainty increases, as the system is better protected against constraint violation. However, the affinely adjustable robust model does not appear very sensitive to the budget of uncertainty.

\section{Conclusions and Future Directions}

In this paper, we investigated a variety of models to incorporate uncertainty in a dynamic pricing and inventory control problem with no stockouts. We summarize the results regarding the complexity of the various formulations in Table 2 .

\begin{tabular}{|c|c|c|c|c|c|c|}
\hline & $\begin{array}{l}\text { open-loop or } \\
\text { closed-loop }\end{array}$ & objective & $\begin{array}{c}\text { number of } \\
\text { variables }\end{array}$ & $\begin{array}{l}\text { number of } \\
\text { constraints }\end{array}$ & $\begin{array}{c}\text { type of } \\
\text { constraints }\end{array}$ & subproblems \\
\hline Deterministic & open-loop & quadratic & $2 N T$ & $(4 N+1) T$ & linear & no \\
\hline Stochastic & open-loop & quadratic & $2 N T$ & $(4 N+1) T$ & $\begin{array}{c}\text { highly } \\
\text { nonlinear }^{a}\end{array}$ & no \\
\hline Robust & open-loop & quadratic & $4 N T$ & $(8 N+1) T$ & linear & no \\
\hline Affine adj. robut & open-loop $^{b}$ & nonlinear & $6 N T$ & $(4 N+1) T$ & nonlinear & yes $^{c}$ \\
\hline $\begin{array}{c}\text { DP } \\
\text { (first stage problem) }\end{array}$ & closed-loop & $\begin{array}{c}\text { highly } \\
\text { nonlinear }^{d}\end{array}$ & $2 N$ & $4 N+1$ & linear & no \\
\hline $\begin{array}{c}\text { Adj. robust } \\
\text { (first stage problem) }\end{array}$ & closed-loop & nonlinear $^{e}$ & $2 N$ & $4 N+1$ & linear & yes \\
\hline
\end{tabular}

${ }^{a}$ actual structure depends on the CDF of the uncertain data

${ }^{b}$ However, the actual decisions do get adjusted depending on realizations

${ }^{c}$ linear constraints, objective is polynomial of degree 2 or 3

${ }^{d}$ actual structure depends on the CDF of the uncertain data; untractable to write the objective in closed-form for multiple time periods

${ }^{e}$ untractable to write the objective in closed-form for multiple time periods

Table 2: Comparison of the models

We showed that robust models allow to efficiently control the trade-off between performance and conservativeness, under limited information, and in this example led to tractable formulations. Stochastic models provide good solutions as long as the distributional assumptions are correct. They may be much more complex to solve though, depending on the probability distribution. The difficulty of solving the robust models essentially depends on the structure of uncertainty sets - i.e. type of budget of uncertainty constraints. Closed-loop solutions clearly perform better than openloop solutions, as they give the possibility to adjust the solution based on current observations. An affine adjustable robust model is a good model to consider as it adapts to demand realizations but does not suffer from the "curse of dimensionality". 
While in this paper, we considered budgets of uncertainty shared, at a give time and for a given product, by the two uncertain demand parameter, there could be other ways to design budget of uncertainty constraints. One of them is to bound the cumulative dispersion over time, for a given parameter and a given product.

$$
\sum_{s=1}^{t}\left|z_{i}(s)\right| \leq \Gamma_{i}(t), \quad \sum_{s=1}^{t}\left|y_{i}(s)\right| \leq \Theta_{i}(t) \quad \forall t, i
$$

where

$$
z_{i}(t) \equiv \frac{\tilde{\alpha}_{i}(t)-\alpha_{i}(t)}{\hat{\alpha}_{i}(t)}, \quad y_{i}(t) \equiv \frac{\tilde{\beta}_{i}(t)-\beta_{i}(t)}{\hat{\beta}_{i}(t)} \in[-1,1]
$$

are the scaled variations of the uncertain parameters around their nominal value and budgets of uncertainty $\Gamma_{i}(),. \Theta_{i}($.$) are increasing with time.$

This model captures that a given parameter is unlikely to take the worst value throughout the time horizon. This budget of uncertainty constraint has the advantage of separating across products. The general notion has been used by several authors (see for example, Bertsimas and Sim [14], Bertsimas and Thiele [15], Adida and Perakis [3, 2]). It may increase the difficulty though, as the budget of uncertainty constraints bind the time instants together, while instantaneous constraints are easier to handle. In the problem under consideration, the constraint would affect only the no stockout constraint. Since the realized inventory is linear with uncertain parameters, the resulting formulation would remain linear.

Another way to design uncertainty sets is to have budget of uncertainty across products, for a given parameter, and at a given time.

$$
\sum_{i=1}^{N}\left|z_{i}(t)\right| \leq \Gamma(t), \quad \sum_{i=1}^{N}\left|y_{i}(t)\right| \leq \Theta(t) \quad \forall t .
$$

This model captures that at a given time, it is most likely that not all products have a parameter at the worst value. The constraint separates across time, but not across products. The resulting robust counterpart may not be easy to formulate as a budget of uncertainty constraint affects multiple constraints from the original problem.

\section{Acknowledgements}

We would like to thank the Associate Editor and Referee of this paper for all the comments that helped us improve the content and exposition of this paper. Preparation of this paper was supported, in part, by award 0556106-CMII from the National Science Foundation and the Singapore MIT Alliance Program. 


\section{References}

[1] E. Adida. Dynamic Pricing and Inventory Control with no Backorder under Uncertainty and Competition. $\mathrm{PhD}$ dissertation, Massachusetts Institute of Technology, Operations Research Center, June 2006.

[2] E. Adida and G. Perakis. A robust optimization approach to dynamic pricing and inventory control with no backorders. Mathematical Programming Special Issue on Robust Optimization, 107(1-2):97-129, June 2006.

[3] E. Adida and G. Perakis. Dynamic pricing and inventory control: Uncertainty and competition. Part A: Existence of a Nash equilibrium. Working paper, Operations Research Center, Massachusetts Institute of Technology, 2007.

[4] V. F. Araman and R. Caldentey. Dynamic pricing for nonperishable products with demand learning. Operations Research, 57(5):1169-1188, 2009.

[5] S. Axsäter and L. Juntti. Comparison of echelon stock and installation stock policies for twolevel inventory systems. International Journal of Production Economics, 45:303-310, 1996.

[6] N. V. Balashevich, R. Gabasov, and F. M. Kirillova. Numerical methods for open-loop and closed-loop optimization of piecewise linear systems. Computational Mathematics and Mathematical Physics, 41(11):1578-1593, 2001.

[7] M. Ben-Daya and A. Raouf. Inventory models involving lead time as a decision variable. The Journal of the Operational Research Society, 45(5):579-582, 1994.

[8] A. Ben-Tal, A. Goryashko, E. Guslitzer, and A. Nemirovski. Adjustable robust solutions of uncertain linear programs. Mathematical Programming, 99(2):351-376, 2004.

[9] A. Ben-Tal and A. Nemirovski. Robust convex optimization. Mathematics of Operations Research, 23:769-805, 1998.

[10] D. P. Bertsekas and J. N. Tsitsiklis. Neuro-Dynamic Programming. Athena Scientific, Belmont, MA, 1996.

[11] D. Bertsimas and S. de Boer. Dynamic pricing and inventory control for multiple products. Journal of Revenue and Pricing Management, 3(4):303-319, January 2005.

[12] D. Bertsimas and A. Mersereau. A learning approach for interactive marketing to a customer segment. Operations Research, 55(6):1120-1135, 2007.

[13] D. Bertsimas and I. Ch. Paschalidis. Probabilistic service level guarantees in make-to-stock manufacturing systems. Operations Research, 49(1):119-133, 2001.

[14] D. Bertsimas and M. Sim. The price of robustness. Operations Research, 52(1):35-53, 2004.

[15] D. Bertsimas and A. Thiele. A robust optimization approach to supply chain management. Operations Research, 54(1), 2006. 
[16] S. Biller, L. M. A. Chan, D. Simchi-Levi, and J. Swann. Dynamic pricing and the directto-customer model in the automotive industry. Electronic Commerce Research, 5(2):309-334, April 2005.

[17] J. R. Birge and F. V. Louveaux. Introduction to Stochastic Programming. Springer, New York, NY, 1997.

[18] G. Bitran and R. Caldentey. An overview of pricing models and revenue management. Manufacturing and Service Operations Management, 5(3):203-229, 2003.

[19] F. Caro and J. Gallien. Dynamic assortment with demand learning for seasonal consumer goods. Management Science, 53(2):276-292, 2007.

[20] L. Chen and E. L. Plambeck. Dynamic inventory management with learning about the demand distribution and substitution probability. Manufacturing and Service Operations Management, 10(2):236-256, 2008.

[21] X. Chen, M. Sim, and P. Sun. A robust optimization perspective of stochastic programming. 2005. Working paper.

[22] X. Chen and D. Simchi-Levi. Coordinating inventory control and pricing strategies with random demand and fixed ordering cost: The finite horizon case. Operations Research, 52(6):887$896,2004$.

[23] X. Chen and D. Simchi-Levi. Coordinating inventory control and pricing strategies with random demand and fixed ordering cost: The infinite horizon case. Mathematics of Operations Research, 29(3):698-723, 2004.

[24] A. J. Clark and H. Scarf. Optimal policies for a multiechelon inventory problem. Management Science, 6:475-490, 1960.

[25] J. Dupačová. On statistical sensitivity analysis in stochastic programming. Annals of Operations Research, 30:199-214, 1991.

[26] L. El-Ghaoui, F. Oustry, and H. Lebret. Robust solutions to uncertain semidefinite programs. SIAM Journal of Optimization, 9(1):33-52, 1999.

[27] W. Elmaghraby and P. Keskinocak. Dynamic pricing: Research overview, current practices and future directions. Management Science, 49:1287-1309, 2003.

[28] G. D. Eppen. Effects of centralization on expected costs in a multi-location newsboy problem. Management Science, 25(5):498-501, 1979.

[29] A. Federgruen and A. Heching. Combined pricing and inventory control under uncertainty. Operations Research, 47:454-475, 1999.

[30] S. M. Gilbert. Coordination of pricing and multiple-period production across multiple constant priced goods. Management Science, 46(12):1602-1616, 2000.

[31] E. Guslitzer. Uncertainty-immunized solutions in linear programming. Master's of science, Technion, Minerva Optimization Center, Faculty of Industrial Engineering and Management, Haifa, Israel, June 2002. 
[32] C. C. Holt, F. Modigliani, J. Muth, and H. A. Simon. Planning Production, Inventories, and Work Force. Prentice-Hall, Inc, Englewood Cliffs, New Jersey, 1960.

[33] S. Kachani and G. Perakis. A fluid model of dynamic pricing and inventory management for make-to-stock manufacturing systems. Working paper, Operations Research Center, Massachusetts Institute of Technology, 2002.

[34] S. Kachani, G. Perakis, and C. Simon. Modeling the transient nature of dynamic pricing with demand learning in a competitive environment. Working paper, Operations Research Center, Massachusetts Institute of Technology, 2005.

[35] C.-J. Liao and C.-H. Shyu. An analytical determination of lead time with normal demand. International Journal of Operations and Production Management, 11(9), 1991.

[36] C. Maglaras and J. Meissner. Dynamic pricing strategies for multi-product revenue management problems. Manufacturing and Service Operations Management, 8(2):136-148, 2006.

[37] I. Ch. Paschalidis and Y. Liu. Pricing in multiservice loss networks: Static pricing, asymptotic optimality, and demand susbtitution effects. IEEE/ACM Transactions On Networking, 10(3):425-438, 2002.

[38] D. Pekelman. Simultaneous price-production decisions. Operations Research, 22:788-794, 1973.

[39] R. Pindyck. Inventories and the short-run dynamics of commodity prices. Working paper, Massachusetts Institute of Technology, 1990.

[40] E. L. Porteus. Stochastic inventory theory. In D. P. Heyman and M. J. Sobel, editors, Handbooks in $O R$ and $M S$, volume 2. Elsevier Science Publishers B.V., Amsterdam, The Netherlands, 1990.

[41] W. Römisch and R. Schultz. Distribution sensitivity in stochastic programming. Mathematical Programming, 50:197-226, 1991.

[42] N. Rudi, S. Kapur, and D. F. Pyke. A two-location inventory model with transshipment and local decision making. Management Science, 47(12):1668-1680, 2001.

[43] N. V. Sahinidis. Optimization under uncertainty: State-of-the-art and opportunities. Computers and Chemical Engineering, 28:971-983, 2004.

[44] A. Senhadji and C. Montenegro. Time series analysis of export demand equations: A crosscountry analysis. IMF Staff Papers, 46:259-273, 1999.

[45] S. P. Sethi, W. Suo, M. Taksar, and H. Yan. Optimal production planning in a multi-product stochastic manufacturing system with long-run average cost. Discrete Event Dynamic Systems: Theory and Applications, 8:37-54, 1998.

[46] M. Slaughter. International trade and labor-demand elasticities. Journal of International Economics, 54:27-56, 2001.

[47] A. Soyster. Convex programming with set-inclusive constraints and applications to inexact linear programming. Operations Research, 21:1154-1157, 1973. 
[48] K. Talluri and G. van Ryzin. The Theory and Practice of Revenue Management. Kluwer Academic Publishers, 2004.

[49] D. H. van Hessem and O. H. Bosgra. Closed-loop stochastic dynamic process optimization under input and state constraints. In Proceedings of the 2002 American Control Conference, 2002 .

[50] H.-J. Zimmerman. Fuzzy Set Theory and its Application. Kluwer Academic Publisher, Boston, second edition, 1991.

[51] P. H. Zipkin. Foundations of Inventory Management. Irwin McGraw-Hill, 2000. 